\title{
Sopra alcune applicazioni degli invariauti adiabatici.
}

\author{
Memoria di Dario Grawfr (a Cagliari).
}

Sunto. - Nel primo capitolo si generalizza e si perfeziona il metodo trattato in ricerche precedenti per verificare se l'errore commesso ritenendo invarianti adiabatici gli integrali ciclici di BOHR-SOMMERFELD di un sistema meccanico qualora i parametri non variano in maniera infinitamente lenta e graduale rimane entro limiti prefissati. I resultati vengono applicati al problema del moto amonico con forza di richiano vaviabile col tempo e al problema dei due corpi di massa variabile dimostrando come una insignificante variazione di eccentricità dell'orbita di uno dei due corpi rispetto all' altro non sia incompatibile con una enorme variazione di massa.

Nel secondo capitolo viene indicata un metodo di integrazione approssimata per* $i$ sistemi meccanici con paranetri dipendenti dal tenpo, e si calcola un valore maggiorante per l'errore commesso con questa approssimazione.

Nel terao capitolo si tratta, in vista di ricesche future, lo studio di un sistena mecanico con due paranetri variabili di cui wno solo in maniera prossima ad essere infinitamente lenta e graduale, e si dimostra come gl' integrali ciclici del sistema possono venire approssimati con altre grandezza di piti facile calcolo.

\section{INTRODUZIONE}

L'esistenza di invarianti adiabatici per un sistema meccanico, dipende anzitutto, come è noto, dal variare infinitamente lento (') di alcuni parametri del sistema stesso. Siccome tale condizione non è mai in pratica verificata esattamente, abbiamo, in note precedenti (2), stabiliti metodi per calcolare se l'errore commesso nelle applicazioni degli invarianti adiabatici resta com. preso entro limiti prefissati. E questi resultati abbiamo applicato al problema dei due corpi con massa variabile.

(1) Come del resto è notissimo non basta ammettere che i parametri varino in maniera infinitamente lenta, ma occorrono altre condizioni per assicurare l'invarianza adiabatica. Ma su questo e sul concetto di variazione infinitamente lenta di un parametro torneremo in seguito.

(2) Gli invarianti adiabatici come metodo d'integrazione approssimata di equazioni differenziali. "Rendiconti Accademia dei Lincei 》, I sem., 1932, pag. 657. Limitazione dei valori degli invarianti adiabatici con applieazione al problema delle masse variabili. Parte I e II "Atti Accademia Torino», 1933. 
Nel presente lavoro, completeremo ed estenderemo quelle ricerche. Oi limiteremo però ancora a sistemi con un grado di libertà, e le grandezze che al limite potrebbero essere invarianti adiabatici, saranno gli integrali ciclici di BOHR-SOMLMEREELD.

Crediamo opportuno per far comprendere meglio lo scopo del lavoro esporre brevemente i principali resultati che in esso verranno conseguiti.

Cominceremo con ottenere, sia pure seguendo metodi già esposti nelle altre note citate, una nuova formula per il limite superiore dell'errore che si commette considerando quegli integrali di SoMMERFELD come invarianti. Queste formule, che sono in sostanza generalizzazione di quelle ottenute in un caso particolare del problema delle masse variabili (1), offrono alouni notevoli vantaggi rispetto alle altre finore ottenute. Infatti tali formule sono di più facile applicazione ai sistemi mecoanici, di più, contenendo grandezze dipendenti dai parametri (e non esplicitamente dal tempo come le formule delle altre note), ossia la variazione (") totale dei parametri stessi in un periodo e nell' intervallo in cui si studia l'eventuale variabilità dell' integrale di SoMmerfeLd, è più spedito ottenere da esse deduzioni generali e di carattere pratico.

Applicheremo questi resultati ai problemi già presi in esame nelle altre note, e cioè al moto armonico con forza d'attrazione dipendente dal tempo e al problema dei due corpi di massa variabile. Nel primo caso otterremo limiti superiori dell' errore commesso in generale più bassi di quelli trovati nella nostra nota citata del 1932. Nel secondo caso potremo generalizzare con opportune ipotesi $i$ resultati che in altri lavori abbiamo ottenuti con leggi speciali per la variazione della massa, cioè verificheremo che una variazione sensibile sull' eccentricità dell' orbita descritta da un corpo rispetto all'altro si può ottenere ammettendo, anzitutto, enormi variazioni della massa dei due corpi.

(1) Sull'ecentricità dell' orbita nel problema dei due corpi di massa variabile. "Rendiconti Accademia dei Lincei ", I sem., 1934, pag. 144 e 223.

(2) Il concetto di variazione totale si trova esposto p. es. in L. TonelLI, Fondamenti di Calcolo delle Variazioni, Bologna 1923, I vol., pag. 40. Siccome in questo trattato a pag. 171 per le funzioni assolutamente continue (e quindi in particolare per le funzioni derivabili e con derivata continua a cai sempre ci limiteremo) si dimostra che la variazione totale di una funzione $f(x)$ in un intervallo $(a, b)$ vale $\int_{a}^{b}\left|f^{\prime}\right| d x$ noi intenderemo sempre questo integrale come la variazione di $f(x)$ in $(a, b)$. i da notare che se $f(x)$ è sempre crescente o decrescente la variazione totale vale $\mid f(b)-f(a)$;. 
Verificheremo inoltre che le formule di questo lavoro sono applicabili anche ad intervalli infiniti, il che non sarebbe possibile con quelle delle altre Note ( $\left.{ }^{1}\right)$.

Passeremo poi a vedere se è possibile ottenere per mezzo del concetto di invariante adiabatico un metodo d'integrazione approssimata delle equazioni del moto di un sistema meccanico, riprendendo così uno studio iniziato nel 1932 e risoluto per il caso particolare del moto armonico ora ricordato. Noi vedremo che questo metodo non è sempre applicabile per tutto l'intervallo in cui si può ritenere l'integrale ciclico invariante con errore trascnrabile, salvo casi molto particolari. Però se limitiamo il metodo d'integrazione ad un intervallo in cai è trascurabile il quadrato della variazione del parametro $\left({ }^{z}\right)$, allora diventa pure trascurabile l'errore commesso nel sostituire la soluzione esatta con quella approssimata. Si noterà che una analoga proprieta vale per il metodo delle perturbazioni, ma a noi sembra il nostro metodo pì̀ semplice, e quello che più importa, è che con questo metodo si può conoscere un limite superiore dell' errore commesso.

Infine svolgeremo la seguente questione che ci si è presentata in un problema di meccanica celeste. Studieremo il caso di un sistema meccanico in eui vi sono due parametri, uno solo però variabile in una maniera prossima a quella che assicura l'invarianza adiabatica degli integrali ciclici. Troveremo che l'integrale ciclico in un intervallo in cui resta finita la variazione totale dei due parametri, si avvicina approssimativamente ad una grandezza di più facile calcolo, almeno in certi casi. O per meglio dire calcoleremo un limite superiore per l'errore che si commette sostituendo all'integrale ciclico questo valore approssimato e vedremo che tale limite diventa tanto più piccolo quanto più la variazione del primo parametro si avvicina a quella che assicura l'invarianza adiabatica. Termineremo con la discussione della portata e dell' applicabilità di questo resultato $\left({ }^{3}\right)$.

(1) Notiamo ehe le formule eitate valgono qualunque sia il modo di variazione dei pa. rametri ma, come vedremo, esse danno errori tanto piu piccoli quanto più il detto modo di variazione si avvicina a quello che assicura l'invarianza adiabatica.

(2) A rigore l'intervallo in oui è valida la nostra integrazione approssimata non coincide con quello indicato. nel testo, ma ne differisce in generale di poco. La cosa sarà naturalmente precisata più avanti.

(3) In un sunto dei miei lavori già citati, fatti dal WINTNer sú « Zentralblatt für Mathematik $\gg(5-15$ e $7-372)$ sỉ afferma che l'idea delle mie altre ricerche non ê nuova, perchè l'ebbe già il KNeser ("Mathematischen Annalen ", 91, 155, 1924). Debbo dichiarare che a me sembra essere scopo del KNeser quello di dare una dimostrazione esatta dell'invarianza adiabatica degli integrali cielici di BoHR-SOMMERFELD, mentre la mia ricerca si propone (ripetiamolo) di ottenere un limite superiore per l'errore commesso, qualora i parametri non varino nel 


\section{CAPTTOTO $\mathrm{I}$.}

1. Formule introduttive. ... Si abbia un sistema meccanico ad un grado di libertà retto dalle equazioni Hamiltoniane nelle variabili $p$ e $q$ :

$$
\frac{d p}{d t}=-\frac{\partial \mathfrak{H}}{\partial q} \quad \frac{d q}{d t}=\frac{\partial \mathscr{H}}{\partial p}
$$

dove la funzione di Hanrlton $\mathscr{H}$ dipende, oltre che da $p$ e $q$, da un certo numero di parametri variabili col tempo. Noi supporremo per fissare le idee che questi parametri siano in numero di due e li indicheremo con le lettere $a$ e $b$. Adoperiamo due parametri in vista delle questioni che tratferemo nel terzo capitolo, sebbene per gli scopi di questo basterebbe usare, (e il procedimento sarebbe più semplice) un parametro solo.

Passiamo a formulare le ipotesi fondamentali pèr la nostra ricerca. Ammetteremo anzitutto che ad ogni valore per $a$ e $b$ compreso entro un certo campo (connesso) $C$ si possa coordinare un campo $A$ del piano delle $p, q$ tale che le soluzioni di (1) con $a$ e $b$ costanti e con condizioni iniziali entro il corrispondente $A$ siano periodiche e tutte contenute in $A$. Potremo percio ad ogni valore $a, b$ di $C$ ed ad ogni $p, q$ contenuto nel corrispondente $A$ coordinare l'integrale ciclico di BoHR-SOMMERFELD $J=\oint p d q$. Queste $J$ relative ai punti di $A$ le supporremo variabili con continuità fra due valori $J_{1}$ e $J_{2}$ alla loro volta dipendenti con continuità da $a, b$. Ammetteremo anche, benchè non sia strettamente necessario, nello spazio delle $J, a, b$, convesso il campo (che diremo campo $B$ ) limitato da $J_{1}, J_{2}$ e da una superficie cilindrica retta di base $C$.

Supporremo poi che si possa esprimere $\mathscr{H}$ come funzione univoca di $J, a, b$, che per ogni $(a, b)$ di $C$ e per ogni $J$ compreso i corrispondenti $J_{1}, J_{2}$, $\frac{\partial \mathcal{H}}{\partial J}$ sia sempre dello stesso segno e che i valori iniziali di $p$ e $q$ siano compresi nel campo $A$ relativo ad $a(0), b(0)$.

modo che assicura l'invarianza adiabatica degli integrali ora citati. Di più nella sua Nota il KNeser non fa aleuna applicazione dei suoi resultati, tanto meno al problema delle masse variabili. nel quale gli invarianti adiabatici furono introdotti solo nel 1928 dal Lev1-Civita. Per queste ragioni mi sembra inesatta l'asserzione del WINTNER.

Debbo dichiarare però che ho preso dal KNESER aleune notazioni.

Si osservi ancora che in questo lavoro si usa l'espressione limite stperiore dell' errore, ma che sarebbe più esatto adoperare il termine valore maggiorante. 
Ciò posto potremo formare l'equazione di $J_{A C O B I}$ ponendo in luogo di $E$ la $\mathscr{H}(J, a, b)$. Mediante un integrale completo di questa equazione $V(q, J, a, b)$ (integrale che si ottiene facilmente mediante una quadratura) potremo avere la nota trasformazione canonica delle variabili $p$ e $q$ in nuove variabili di cui una e la $J$ e l'altra la sua coniugata che indicheremo con $-w$. Tale trasformazione sarà data ovviamente dalle equazioni:

$$
p=\frac{\partial V}{\partial q} \quad-w=-\frac{\partial V}{\partial J}
$$

le quali come è noto definiscono $p$ e $q$ come funzioni periodiche di $w$ con periodo 1 s'intende per $(a, b)$ costante e entro $C$ e $J$ compreso fra i corrispondenti $J_{1}, J_{2}$.

Allora le equazioni relative alle $J$ e $v$ saranno sempre del tipo Hamiltoniano con la funzione di HaмıLToN uguale alla $H(J, a, b)+\frac{\partial V}{\partial t}$

$$
\frac{d J}{d t}=\frac{\partial}{\partial(-w)}\left(\frac{\partial V}{\partial t}\right) \quad \frac{d(-w)}{d t}=-\left(\frac{\partial H}{\partial J}+\frac{\partial}{\partial J}\left(\frac{\partial V}{\partial t}\right)\right)
$$

Ora la $V$ è funzione esplicita del tempo solo attraverso $a$ e $b$ perciò $\dot{e}$ :

$$
\frac{\partial V}{\partial t}=\frac{\partial V}{\partial a} a^{\prime}+\frac{\partial V}{\partial b} b^{\prime}
$$

Se ora indichiamo con $J_{a}, J_{b}, w_{a}, w_{b}$, $\omega$ rispettivamente le derivate $\frac{\partial^{2} V}{\partial m \partial a}, \frac{\partial^{2} V}{\partial w \partial b}, \frac{\partial^{2} V}{\partial J \partial a}, \frac{\partial^{2} V}{\partial J \partial b}, \frac{\partial H}{\partial J}$, le (3) assumono la forma:

$$
\left\{\begin{array}{l}
\frac{d J}{d t}=-a^{\prime} J_{a}-b^{\prime} J_{b} \\
\frac{d w}{d t}=\omega+a^{\prime} w_{a}+b^{\prime} w_{b} .
\end{array}\right.
$$

Le $J_{a}, J_{b}, w_{a}, w_{b}$ saranno in generale funzioni di $J, w, a, b$. Esse godono anzitutto della seguente proprietà. Essendo come è noto $\frac{\partial V}{\partial a}, \frac{\partial V}{\partial b}$. ( $\left.{ }^{1}\right)$, fissati

(1) Per comprendere ciò si osservi che esprimendo la $V(q, J, a, b)$ in funzione di $w$ cioè ponendo al posto $q$ la sua espressione in funzione di $w$ si ottiene una funzione multiforme tale cioè che se $w$ aumenta di uno la $V$ aumenta di $J$ ossia si ha:

$(x)$

$$
V(q(w+1), J, a, b)=V(q(w v), J, a, b)+J
$$

E questa relazione è immediata conseguenza dell' altra:

$$
V(q(w+1), J, a, b)-V(q(w), J, a, b)=\oint p d q=J
$$


$a, b$ e $J$ (s'intende $(a . b)$ entro $C, J$ fra $J_{1}$ e $J_{2}$ ) funzioni periodiche di $w$ con periodo 1 saranno tali anche $J_{a}, J_{b}, w_{a}, w_{b}$. Di più sarà :

$$
\int_{w}^{n+1} J_{a} d w=\int_{w}^{w+1} \frac{\partial^{2} V}{\partial w \partial a} d w=\left[\frac{\partial V}{\partial a}\right]_{w}^{w+1}=0
$$

e analogamente:

$$
\int_{w}^{w+1} J_{b} d w=0
$$

Ammetteremo poi che le $\omega, J_{a}, J_{b}, w_{a}, w_{b}$ siano per ogni $a, b$ in $C$ e per ogni $J$ e $w$ comprese rispettivamente fra $J_{1}$ e $J_{2}, w^{\prime}, w^{\prime}+2\left(w^{\prime}\right.$ numero reale qualunque) sempre limitate e a rapporto incrementale limitato rispetto a tutte quattro le variabili, $a, b, J, w$. 亡̀ ovvio, per la periodicità delle nostre funzioni, che queste ipotesi valgono per ogni $w$. ferme restando i limiti per $a, b$ e per $J$. Ovviamente poi le equazioni (5) e tutte le loro proprietà sono valide fino a che dalle $J$ e $w$ che si ottengono risolvendo tali equazioni corrispondono per le (2) valori di $p$ e $q$ compresi entro $C$ cioè fino a che $J(t)$ resta compresa fra le $J_{1}$ e $J_{2}$ corrispondenti ad $a(t), b(t)$.

A questo punto, sarà bene notare che le ipotesi che abbiamo ora ricordate si potrebbero dedurre da altre più semplici fatte sulle equazioni (1). Abbiamo creduto opportuno non seguire questa via che ci porterebbe troppo lontano.

Passiamo ora ad alcune definizioni relative ai parametri. Ripetiamo che per variazione totale $V(a, t)$ del parametro $a$ in un intervallo $(0, t)$ intenderemo l'espressione:

$$
V(\alpha, t)=\int_{0}^{t}\left|\frac{d a}{d t}\right| d t
$$

Derivando ora la $(\varkappa)$ rispetto ad a avremo ricordando che la $\frac{\partial V}{\partial a}$ che compare nelle nostre formule si ottiene derivando $V$ mantenendo costante $q$ si ha:

$$
\left\{\frac{\partial V}{\partial q} \frac{\partial q}{\partial a}\right\}_{w+1}+\left\{\frac{\partial V}{\partial a}\right\}_{w+1}=\left\{\frac{\partial V}{\partial q} \frac{\partial q}{\partial a}\right\}_{w}+\left\{\frac{\partial V}{\partial a}\right\}_{w}
$$

dove nel primo membro $i$ termini si suppongono calcolati in $w+1$ nel secondo membro in $w$ a parita s'intende di $a, b, J$. Ora $\frac{\partial V}{\partial q}=p$ è funzione periodica di periodo 1 di $w$ così pure sarà $\frac{\partial q}{\partial a}$ essendo periodica di periodo 1 per ogni $a, b$ in $C$ la $q$. Si ha allora che il primo termine al primo membro di (B) si elide col primo termine a secondo membro restando così:

$$
\left\{\frac{\partial V}{\partial a}\right\}_{w+1}=\left\{\frac{\partial V}{\partial a}\right\}_{n}
$$

e cio dimostra la periodicità di $\frac{\partial V}{\partial a}$ rispetto a $w$. Annloga dimostrazione vale pei $\frac{\partial V}{\partial b}$. 
che se $a$ è sempre crescente o decrescente in $(0, t)$ diviene:

$$
V(a, t)=|a(t)-a(0)| \text {. }
$$

Se invece $a$ non è sempre crescente o decrescente ma ha in $(0, t)$ un numero finito di massimi o minimi l'espressione di $V(a, t)$ non è più così semplice, ma il calcolo di essa non è affatto difficile.

Conviene infatti per eseguire questo calcolo, dividere l'intervallo $(0, t)$ in un certo numero di tratti in cui $a(t)$ è sempre crescente o decrescente ed applicare a questi tratti la formula (9). Analoghe definizioni valgono per il parametro $b$.

Intenderemo poi per periodo del moto, relativo all' istante $t$, il periodo del moto retto dalle (1) con $J, a, b$ costanti e aguali a $J(t), a(t), b(t)$ e con $\mathscr{H}$ uguale al valore corrispondente a $J(t), a(t), b(t)$. Questo periodo $T(t)$ vale ovviamente $\frac{1}{\omega(J(t): a(t), b(t))}\left({ }^{1}\right)$.

Diremo perciò variazione del parametro $a$ o $b$ nel periodo $T$ corrispondente all'istante $t$ (grandezza che indicheremo.con $\Delta_{t} a, \Delta_{t} b$ o se non vi è luogo ad equivoco con $\Delta a, \Delta b)$ la variazione di questo parametro nel periodo $t, t+T(t)$. Sarà cioè :

$$
\Delta_{t} a=\int_{t}^{t+T(t)}\left|\frac{d a}{d t}\right| d t \quad \Delta_{t} b=\int_{t}^{t+T(t)}\left|\frac{d b}{d t}\right| d t .
$$

Se la variazione di un parametro per ogni valore di $t$ di un certo intervallo $(0, h)$ si può considerare come infinitesima diremo che il parametro varia in $(0, h)$ in maniera infinitamente lenta. Infine diremo (seguendo in sostanza il Levi-Orvita) ohe un parametro $a$ o $b$ varia in $(0, h)$ in maniera graduale quando si può considerare come infinitesimo per ogni $0<t<h$ la variazione totale delle sue derivate in $t, t+T(t)$ rispetto al valore assoluto della derivata stessa calcolata nel punto $\bar{t}$ intermedio fra $t$ e $t+T(t)$ e tale che $\left|a^{\prime}(\bar{t})\right| T(t)$ o $\left|b^{\prime}(\bar{t})\right| T(t)$ valga $\Delta_{t} a$ o $\Delta_{t} b$. Cioè : affinchè il parametro $a$ vari in maniera graduale deve essere infinitesima l' espressione che indicheremo con $\Delta_{t} \alpha^{\prime}$

$$
\Delta_{t} a^{\prime}=\frac{\int_{t}^{t-T}\left|\frac{d^{2} a}{d t^{2}}\right| d t}{\left|\frac{d a}{d t}\right|_{t+\theta T}}
$$

in cui $t+\theta T=\bar{t}$. Analoga definizione si può dare per $\Delta_{t} b^{\prime}$.

(i) Si noti che in seguito ammetteremo w positiva. Se ciò non fosse si può sempre ridursi a questo caso cambiando $t$ in - $t$. 
2. Calcolo di un fondamentale limite superioce. - Consideriamo l'equazione differenziale:

$$
\frac{d J}{d t}=-a^{\prime} J_{a}-b^{\prime} J_{b}
$$

e l'altra equazione differenziale nella $y$

$$
\frac{d y}{d t}-b^{\prime} \bar{J}_{b}
$$

Dove $\bar{J}_{b}$ non è altro che $J_{b}$ in cui però in luogo di $J$ si è messo la $y$ gran* dezza che ora preciseremo. Noi ci proponiamo di calcolare un intervallo entro cui la differenza fra $J$ e $y$ resta in modulo inferiore ad un numero $\eta$ positivo prefissato, ma tale che in un certo intervallo $\left(0, h^{\prime}\right), y(t)+\eta$ sia sempre inferiore al corrispondente $J_{2}, y(t)-\eta$ superiore al corrispondente $J_{1}\left({ }^{i}\right)$. La $y$ è poi definita oltre che dell' equazione (13) anche dalla condizione:

$$
y(0)=J(0)=J_{0}
$$

la quale per la continuità di $J_{1}$ e $J_{z}$ e per il fatto che $J_{0}$ è compreso fra la $J_{1}$ e $J_{z}$ corrispondente a $a(0), b(0)$, assicura I'esistenza di $\left(0, h^{\prime}\right)$.

Ora per ragioni di continuità esisterà evidentemente un certo intervallo intorno all' origine $(0, h)$ compreso in $\left(0, h^{\prime}\right)$ in cui $|J-y| \leq \eta$. Ciò assicura che in $(0, h), J$ è compreso fra $J_{i} \otimes J_{2}$ e perciò sono applicabili tutte le proprietà ammesse per il sistema (5). Allora sottraendo membro a membro. la (13) dalla (12) e integrando l'espressione ottenuta da 0 a $t^{\prime}\left(t^{\prime} \leq h\right)$ abbiamo:

$$
|J-y| \leq\left|\int_{0}^{t^{\prime}} a^{\prime} J_{a} d t\right|+\left|\int_{0}^{t^{\prime}} b^{\prime}\left(J_{b}-\bar{J}_{b}\right) d t\right|
$$

Proponiamoci dunque di calcolare un limite superiore $I\left(t^{\prime}\right)$ di $\left|\int_{0}^{t^{\prime}} \alpha^{\prime} J_{a} d t\right|$.

A questo scopo, mediante i punti $t_{i}=\frac{1}{\omega(\mathcal{J}(0), a(0), b(0))}$

$$
\begin{gathered}
t_{2}=t_{1}+\frac{1}{\omega\left(J\left(t_{1}\right), a\left(t_{1}\right), b\left(t_{1}\right)\right)} \cdots t_{r}=\frac{1}{\omega\left(J\left(t_{r-1}\right), a\left(t_{n-1}\right), b\left(t_{r-1}\right)\right.}+t_{r-1} \\
t_{n-1}=t_{n-2}+\frac{1}{\omega\left(J\left(t_{n-2}\right), a\left(t_{n-2}\right), b\left(t_{n-2}\right)\right)}
\end{gathered}
$$

(1) S'intende che $\left(O, h^{\prime}\right)$ deve essere tale che in esso $a(t)$ e $b(t)$ siano entro $C$. 
dividiamo l'intervallo $(0, t)$ in $n$ parti, supponendo che:

$$
t_{n}=t_{n-1}+\frac{1}{\omega\left(J\left(t_{n-1}\right), a\left(t_{n-1}\right) \cdot b\left(t_{n-1}\right)\right)}
$$

sia superiore a $t^{\prime}$. Allora avremo:

$$
\left|\int_{0}^{t} a^{\prime} J_{a} d t\right| \leq I_{01}+I_{12} \ldots I_{n, v+1} \ldots+\left|\int_{t_{n-1}}^{t} \alpha^{\prime} J_{a} d t\right|
$$

dove:

$$
I_{r, r+1}=\left|\int_{t_{r}}^{t_{r+1}} \alpha^{\prime} J_{a} d t\right| .
$$

Calcoliamo ora un limite superiore per $I_{r, r_{+1}}$. A questo scopo consideriamo la funzione, $J_{a}^{\prime}$ ottenuta ponendo in $J_{a}$ al posto di $J(t), a(t), \omega(t), J\left(t_{r}\right), a\left(t_{\eta}\right)$, $w_{1}(t)$ essendo $w_{1}(t)$ uguale a $w\left(t_{r}\right)+\frac{t-t_{r}}{t_{r+1}-t_{r}}$. Potremo scrivere:

$$
I_{r, r+1} \leq\left|\int_{t_{r}}^{t_{r+1}} \alpha^{\prime}\left(J_{a}-J_{a}^{\prime}\right) d t\right|+\left|\int_{t_{r}}^{t_{r+1}} a^{\prime} J_{a}^{\prime} d t\right| .
$$

Ora dalla prima di. (5) si ha per l'intervallo $\left(t_{r}, t_{r+1}\right)$

$$
\begin{gathered}
\left|J(t)-J\left(t_{r}\right)\right|=\left|\int_{t_{r}}^{t} \alpha^{\prime} J_{a} d t+\int_{t_{r}}^{t} b^{\prime} J_{b} d t\right| \\
\leq M_{a} \int_{t_{r}}^{t}\left|a^{\prime}\right| d t+M_{b} \int_{t_{r}}^{t}\left|b^{\prime}\right| d t .
\end{gathered}
$$

Dove $M_{a}$ e $M_{b}$ sono rispettivamente i limiti superiori di $\left|J_{a}\right|$ e $\left|J_{b}\right|$ per ogni $a(t)$ e $b(t)$ con $t$ variabile in $\left(0, t^{\prime}\right)$, per qualunque $w$ e per $J$ compreso fra il massimo di $y(t)+\eta$ e il minimo di $y(t)-\eta$ s'intende in $\left(0, t^{\prime}\right)\left({ }^{1}\right)$. Le $M_{a}$, $M_{b}$ sono finite per le ipotesi fatte sulle limitazioni di $J_{a}, J_{b}$.

(1) Puo darsi che qualche $J, a, b$ compresi fra i limiti ora indicati cada fuori del campo $B$ in cui sono definite le $J_{n}, J_{b}$. Siccome però i valori di queste grandezze che com. paiono nelle nostre equazioni sono calcolate per $J, a, b$ entro $B$, noi intenderemo che $M_{t}$ e $M_{b}$ sono $i$ limiti superiori di $\left|J_{a}\right|$ e $\left|J_{l}\right|$ per quei valori di $d, a, b$ compresi fra $i$ limiti precisati nel testo e interni a $B$. $O$ in altre parole potremo convenire ohe le $J_{a}$ e $J_{b}$ per valori di $J, a, b$ fuori di $B$ sono nulle. Queste avvertenze valgono anche con le ovvie mo. dificazioni per easi analoghi che incontreremo in seguito. 
D'altra parte abbiamo dalla seconda delle (5):

$$
w(t)=\int_{t_{r}}^{t} \omega(J, a, b) d t+\int_{t_{r}}^{t} a^{\prime} w_{a} d t+\int_{t_{r}}^{t} b^{\prime} w_{b} d t+w\left(t_{r}\right) .
$$

Perciò si ha subito:

$$
\begin{aligned}
\left|w(t)-w_{1}(t)\right| \leq & \int_{t_{r}}^{t}\left|\omega(J, a, b)-\omega\left(J\left(t_{r}\right), a\left(t_{r}\right), b(t,)\right)\right| d t \\
& +\left|\int_{t_{r}}^{t} a^{\prime} w_{a} d t\right|+\left|\int_{t_{r}}^{t} b^{\prime} w_{b} d t\right| .
\end{aligned}
$$

E poichè si suppone la $\omega$ Lipschitziana, esisteranno tre numeri positivi $H$, $K_{a}, K_{b}$ tali che:

$$
\begin{gathered}
\left|m(J, a, b)-\omega\left(J\left(t_{r}\right), a\left(t_{r}\right), b\left(t_{,}\right)\right)\right| \leq \\
\leq H\left|J(t)-J\left(t_{r}\right)\right|+K_{a}\left|a(t)-a\left(t_{r}\right)\right|+ \\
+K_{b}\left|b(t)-b\left(t_{r}\right)\right| \leq\left(M_{a} H+K_{a}\right) \int_{t_{r}}\left|a^{\prime}\right| d t+\left(M_{b} H+K_{b}\right) \int_{t_{r}}^{t}\left|b^{\prime}\right| d t .
\end{gathered}
$$

Le $H, K_{a}, K_{b}$ sono prese relativamente a tutti i valori di $t$ (') compresi fra $t_{r}$ e $t_{r^{+}+1}$ e a tutti i valori di $J$ compresi fra il massimo e il minimo di $J(t)$ in $\left(t_{1}, t_{r+1}\right)$.

Se ora sostituiamo nella (20) la (21) e indichiamo con $A$ e $B$ i valori massimi di $\left|w_{a}\right|,\left|w_{b}\right|$ per ogni $w$, per ogni $t$ di $\left(0, t^{\prime}\right)$ e per $J$ compreso fra il massimo di $Y+\eta$ e il minimo di $Y-\eta$ in $\left(0, t^{\prime}\right)$, abbiamo:

$$
\begin{gathered}
\left|w(t)-w_{1}(t)\right| \leq \int_{t_{r}}^{t} d t\left(M_{a} H+K_{a}\right) \int_{t_{r}}^{t}\left|a^{\prime}\right| d t+ \\
+\int_{t_{r}}^{t} d t\left(M_{b} H+K_{b}\right) \int_{t_{k}}^{t}\left|b^{\prime}\right| d t+A \int_{t_{r}}^{t}\left|a^{\prime}\right| d t+B \int_{t_{r}}^{t}\left|b^{\prime}\right| d t .
\end{gathered}
$$

Ciò posto torniamo alla (17). Poichè $J_{a}$ è per ipotesi Lipschitziana potremo trovare quattro numeri positivi $R, S, P, Q$ tali che per ogni $w$ e per

(1) Più precisamente di $a(t), b(t)$ per $t$ compreso ecc.. In seguito useremo spesso la locuzione abbreviata del testo. 
valori di $J$ e $t$ compresi fra gli stessi limiti indicati a proposito, di $H, K_{a}, K_{\iota}$ sia:

$$
\begin{gathered}
\left|J_{a}-J_{a}^{\prime}\right| \leq R\left|J(t)-J\left(t_{r}\right)\right|+S\left|w(t)-w_{1}(t)\right|+ \\
+P\left|a(t)-a\left(t_{r}\right)\right|+Q\left|b(t)-b\left(t_{r}\right)\right| .
\end{gathered}
$$

Abbiamo allora ricordando la (18) e la (21):

$$
I_{r, r+1} \leq R \int_{t_{r}}^{t_{r+1}}\left|a^{\prime}\right| M_{\alpha} d t \int_{t_{r}}^{t}\left|a^{\prime}\right| d t+R \int_{t_{r}}^{t_{r+1}} d t M_{b}\left|a^{\prime}\right| \int_{t_{r}}^{t}\left|b^{\prime}\right| d t
$$

$$
\begin{aligned}
& +S \int_{t_{r .}}^{t_{r+1}}\left|a^{\prime}\right|\left(M_{a} H+K_{a}\right) d t \int_{t_{r}}^{t} d t \int_{t_{r}}^{t}\left|a^{\prime}\right| d t+S \int_{t_{r}}^{t_{r+1}}\left|a^{\prime}\right| d t\left(M_{b} H+K_{b}\right) \int_{t_{r}}^{t} d t \int_{t_{r}}^{t}\left|b^{\prime}\right| d t \\
& +S A \int_{t_{r}}^{t_{r+1}}\left|a^{\prime}\right| d t \int_{t_{r}}^{t}\left|a^{\prime}\right| d t+S B \int_{t_{r}}^{t_{r+1}}\left|a^{\prime}\right| d t \int_{t_{r}}^{t}\left|b^{\prime}\right| d t \\
& +P \int_{t_{r}}^{t_{r+1}}\left|a^{\prime}\right| d t \int_{t_{r}}^{t}\left|a^{\prime}\right| d t+Q \int_{t_{r}}^{t_{r+1}}\left|a^{\prime}\right| d t \int_{t_{r}}^{t}\left|b^{\prime}\right| d t \\
& +\left|\int_{t_{r}}^{t_{r+1}} a^{\prime} J_{a^{\prime}} d t\right|
\end{aligned}
$$

Ora si ha subito:

$$
\begin{gathered}
\int_{t_{r}}^{t_{r+1}+1}\left|a^{\prime}\right| \int_{t_{r}}^{t}\left|a^{\prime}\right| d t=\frac{1}{2}\left(\int_{t_{r}}^{t_{r+1}}\left|a^{\prime}\right| d t\right)^{2} \\
\iint_{t_{r}}^{t_{r+1}}\left|a^{\prime}\right| d t \int_{t_{r}}^{t}\left|b^{\prime}\right| d t \leq \int_{t_{r}}^{t_{r+1}}\left|a^{\prime}\right| d t \int_{t_{r}}^{t_{r+1}}\left|b^{\prime}\right| d t \\
\int_{t_{r}}^{t_{r+1}}\left|a^{\prime}\right| d t \int_{t_{r}}^{t} d t \int_{t_{r}}^{t}\left|a^{\prime}\right| d t \leq \int_{t_{r}}^{t_{r+1}}\left|a^{\prime}\right| \int_{t_{r}}^{t}\left|a^{\prime}\right| d t\left(t-t_{r}\right) \leq \frac{t_{r+1}-t_{r}}{2}\left(\int_{t_{r}}^{t_{r+1}}\left|a^{\prime}\right| d t\right)^{2} \\
\int_{t_{r}}^{t}\left|a^{\prime}\right| d t \int_{t_{r}}^{t} d t \int_{t_{r}}^{t}\left|b^{\prime}\right| d t \leq \int_{t_{r}}^{t_{r+1}}\left|a^{\prime}\right| d t \int_{t_{r}}^{t}\left|b^{\prime}\right| d t\left(t-t_{r}\right) \leq\left(t_{r+1}-t_{r}\right) \int_{t_{r}}^{t_{r}}\left|a^{\prime}\right| d t \int_{t_{r}}^{t_{r+1}}\left|b^{\prime}\right| d t .
\end{gathered}
$$

Abbiamo poi con una semplice integrazione per parti:

$$
\int_{t_{r}}^{t_{r+1}} a^{\prime} J_{a}^{\prime} d t=a^{\prime}\left(t_{r+1+1}\right) \int_{t_{r}}^{t_{r+1}} J_{a}^{\prime} d t-\int_{t_{r}}^{t_{r+1}+1} a^{\prime \prime} \int_{t_{r}}^{t} J_{a}^{\prime} d t
$$


Ora $J_{a}^{\prime}$ non $\grave{e}$ altro che la $J_{a}$ in cui sono fissati i valori di $J, a, b$ e solo la $w_{1}$ è variabile. Di più la $w_{1}$ per $t=t_{r}$ vale $w\left(t_{r}\right)$ per $t=t_{r+1}$ vale $w\left(t_{v}\right)+1$. Si ha perciò ricordando la $(7)$

Quindi dalla (26) si ha:

$$
\int_{t_{r}}^{t_{r+1}} J_{a^{\prime}} d t=\frac{1}{\omega} \int_{w_{1}\left(t_{r}\right)}^{w_{1}\left(t_{r}\right)+1} J_{a}^{\prime} d w_{\mathrm{t}}=0
$$

$$
\left|\int_{t_{r}}^{t_{r+1}} a^{\prime} J_{a}^{\prime} d t\right| \leq M_{a}\left(t_{r+1}-t_{r}\right) \int_{t_{r}}^{t_{r+1}}\left|a^{\prime \prime}\right| d t \leq \frac{M_{a} \int_{t_{r}}^{t_{r+1}}\left|a^{\prime \prime}\right| d t \int_{t_{r}}^{t_{r+1}}\left|a^{\prime}\right| d t}{\left|\bar{a}^{\prime}\right|} .
$$

Essendo $\left|\bar{a}^{\prime}\right|$ quel valore di $\left|a^{\prime}\right|$ compreso fra $t_{r}$ e $t_{r^{\prime}+1}$ tale che

$$
\left|\overline{a^{\prime}}\right|\left(t_{r+1}-t_{,}\right)=\int_{t_{r}}^{t_{r+1}}\left|a^{\prime}\right| d t .
$$

Percio sostituendo nella (24) abbiamo:

$$
I_{r, r+1} \leq Z_{r}\left(\Delta t_{r} a\right)^{2}+Z_{r}{ }^{\prime}\left(\Delta t_{q} b\right)\left(\Delta t_{r} a\right)+M_{a} \Delta_{t_{r}} a^{\prime} \Delta t_{r} a .
$$

Dove:

$$
\begin{aligned}
& Z_{r}=\frac{R M_{a}+S\left(M_{a} H+K_{a}\right)\left(t_{r+1}-t_{r}\right)+S A+P}{2} \\
& Z_{r}{ }^{\prime}=R M_{b}+S\left(M_{b} H+K_{b}\right)\left(t_{r+1}-t_{r}\right)+S B+Q .
\end{aligned}
$$

D'altra parte si ha:

$$
\left|\int_{t_{n-1}}^{t^{\prime}} \alpha^{\prime} J_{a} d t\right| \leq M_{a} \int_{t_{n-1}}^{t^{\prime}}\left|a^{\prime}\right| d t \leq M_{a} \Delta t_{n-1} a
$$

Indichiamo ora con $Z$ e $Z^{\prime}$ due numeri superiori rispettivamente a tutti i $Z_{r}$ e $Z_{r}^{\prime}$. Siano poi $\Delta_{m} a$ e $\Delta_{m} a^{\prime}$ i valori massimi in $\left(0, t^{\prime}\right)$ di $\Delta_{t} a$ e $\Delta_{t_{r}} a^{\prime}$ per ogni $t$ di $\left(0, t^{\prime}\right)$. Allora ricordando, che $V\left(a, t^{\prime}\right)>V\left(a, t_{n-1}\right), V\left(b, t^{\prime}\right)>V\left(b, t_{n-1}\right)$ si ha sostituendo nella (15) i limiti superiori ora ottenuti:

$$
\begin{aligned}
\left|\int_{0}^{t^{\prime}} a^{\prime} J_{a} d t\right| & \leq Z \Delta_{m} a V\left(a, t^{\prime}\right)+Z^{\prime} \Delta_{m} a V\left(b, t^{\prime}\right) \\
& +M_{a} \Delta_{m} a^{\prime} V\left(a, t^{\prime}\right)+M_{a} \Delta_{m} a .
\end{aligned}
$$

Il secondo membro di (32) è il cercato limite superiore che verrà indicato con $I\left(l^{\prime}\right)$. 
Prima di chiudere questo paragrafo aggiungiamo la seguente osservazione. Si consideri una funzione $g$ di $J, g=f(J)$ funzione tale che $f^{\prime}(J)$ sia sempre positiva o sempre negativa, quando $J$ varia fra il massimo valore di $J_{2}$ e il minimo valore di $J_{1}$ relativo all'intervallo $\left(0, h^{\prime}\right)$. Allora si potrà invertire questa relazione e scrivere $J=\varphi(g)$. Ciò permette di scrivere le nostre equazioni in modo da avere come incognite la $g$. Si ponga infatti nelle $\omega, J_{a}, J_{b}, w_{a}, w_{b}$ delle $(5)$ in luogo di $J$ la $\varphi(g)$. Poi si moltiplichi la prima delle (5) per $f^{\prime}(J)=f^{\prime}(\varphi(g))$. Si ottiene così :

$$
\frac{d g}{d t}=-a^{\prime} f^{\prime}(\varphi(g)) J_{a}-b^{\prime} f^{\prime}(\varphi(g)) J_{b}, \quad \frac{d w}{d t}=\omega+a^{\prime} w_{a}+b^{\prime} w_{b}
$$

Ora se si suppone $f^{\prime}$ e $\varphi$ limitate e Lipschitziane si ottiene subito che i termini moltiplicati per $\alpha^{\prime}, b^{\prime}$ al secondo membro delle equazioni ora scritte godono delle stesse proprietà di $J_{a}, J_{b}$ e $w_{a}, w_{b}$ e lo stesso può dirsi della $\omega$.

Perciò i resultati ora ottenuti per $J$ valgono anche per $g$ naturalmente tenendo conto dei diversi valori che ora assumono i secondi membri delle equazioni che definiscono $w$ e $g$.

3. Regole per il calcolo pratico della espressione $\boldsymbol{I}(\boldsymbol{t})$. - Nella (32) compare la $Z, Z^{\prime}, M_{a}, \Delta_{m} \alpha, \Delta_{m} \alpha^{\prime}$ perciò dobbiamo anzitutto indicare qualche metodo per il calcolo di queste grandezze in un intervallo $\left(0, t^{\prime}\right)$ inferiore a $(0, h)$.

Si osservi anzitutto che $M_{a}, M_{b}$ sono quantità valide in base alla loro definizione per qualunque $r$ e si calcolano trovando il massimo valore di $J_{a}, J_{b}$ per $t$ in $\left(0, t^{\prime}\right)$ per ogni $w$ e $J$ compreso fra il valore massimo in $\left(0, t^{\prime}\right)$ di $y \dashv-\eta$ e il valore minimo sempre in $\left(0, t^{\prime}\right)$ di $y-\eta$ valori che indicheremo rispettivamente con $y_{\max }+\eta$ e $y_{\min }-\eta$.

Passiamo ora alle $Z$. Perciò prendiamo in esame i termini che compaiono nelle $Z_{r}$. In esse vi sono anzitutto le $A, B$ indipendenti da $r$ perchè valgono rispettivamente $\mathrm{i}$ limiti superiori $\mathrm{di}\left|w_{a}\right|,\left|w_{b}\right|$ per $t$ in $\left(0, t^{\prime}\right)$ e compreso fra $y_{\max }+\eta, y_{\min }-\eta$ e per ogni $w$. Le $R, S, P, Q$ definite dalle (23) e che compaiono nella espressione di $Z_{0}$. devono coincidere (almeno sotto condizioni di derivabilità per $J_{a}, J_{b}$ che supporemo largamente soddisfatte) rispettivamente con un valore assoluto di $\frac{\partial J_{a}}{\partial J}, \frac{\partial J_{a}}{\partial w}, \frac{\partial J_{a}}{\partial \alpha}, \frac{\partial J_{a}}{\partial b}$ nel campo definito da $-\infty<w<+\infty t_{r}<t<t_{r+1}\left({ }^{1}\right), J$ compreso fra il massimo e il minimo

(1) O meglio fra $a(t), b(t)$ comprese rispettivamente fra $i$ loro massimi o nimimi in $\left(t_{r}, t_{r+1}\right)$ 
di $J(t)$ in $\left(t_{r}, t_{r+1}\right)\left({ }^{1}\right)$. Analogamente $H, K_{a}, K_{b}$ non sono altro che valori assoluti di $\frac{\partial \omega}{\partial J}, \frac{\partial \omega}{\partial a}, \frac{\partial \omega}{\partial b}$ calcolati in un punto del campo ora descritto. Infine $t_{r+1}-t_{r}$ non è altro che $\frac{1}{\omega\left(J\left(t_{r}\right), a\left(t_{r}\right), b\left(t_{r}\right)\right)}$. Ora il calcolo esatto di queste grandezze è impossibile perchè bisognerebbe conoscere $J(t)$ e $w(t)$ ehe sono incognite. Se ne può calcolare un limite superiore prendendo il valore massimo di queste grandezze per ogni $t$ di $\left(0, t^{\prime}\right)$ per ogni $w$, e per ogni $J$ compreso fra $y_{\max }+\eta, y_{\min }-\eta$. Ponendo queste grandezze nelle espressioni di $Z_{r}$. e $Z_{r}^{\prime}$ si troverebbe un valore superiore a qualunque $Z_{r}$. e $Z_{r}^{\prime}$ e questo valore si potrebbe prendere rispettivamente per $Z$ e $Z^{\prime}$.

Vi è però la possibilità di ottenere valori più bassi di $Z$ e $Z^{\prime}$ in base alle seguenti osservazioni che faremo riferendoci a $Z$ perchè analogamente si può procedere per $Z^{\prime}$.

In $Z$ intervengono le espressioni $H\left(t_{r^{+}+1}-t_{r}\right), K_{a}\left(t_{p+1}-t_{v}\right)$. Ora $H$ è un valore di $\left|\frac{\partial \omega}{\partial J}\right|$ calcolato per $t$ compreso fra $t$, e $t_{r^{+}+1}$ e per $J$ compreso fra il massimo e il minimo di $J(t)$ in $t_{r}, t_{r+1}$. Perciò si ha $\left(^{2}\right)$ :

$$
H=\left\{\left(\frac{\partial \omega}{\partial J}\right)_{t_{r}}+\left\{\frac{\partial^{2} \omega}{\partial J^{2}}\right\}\left(J-J\left(t_{r}\right)\right)+\left\{\frac{\partial^{2} \omega}{\partial J \partial a}\right\}\left(a(t)-a\left(t_{r}\right)\right)+\left\{\frac{\partial^{2} \omega}{\partial J \mathcal{E} b}\right\}\left(b(t)-b\left(t_{r}\right)\right) \mid .\right.
$$

Dove $\left\{\frac{\partial \omega}{\partial J}\right\}_{t_{r}}$ è il valore di $\frac{\partial \omega}{\partial J}$ calcolato in $t$. mentre $\frac{\partial^{z} \omega}{\partial J^{2}}, \frac{\partial^{2} \omega}{\partial J \partial a}, \frac{\partial^{z} \omega}{\partial J \partial b}$ sono valori di queste derivate calcolate per $J, a$ e $b$ nel campo descritto a proposito di $H$. Quindi ricordando che $\left|J-J\left(t_{v}\right)\right|<M_{a} \Delta_{m} \alpha+M_{b} \Delta_{m} b$, $\left|a-a\left(t_{r}\right)\right|<\Delta_{m} a,\left|b-b\left(t_{v}\right)\right|<\Delta_{m} b$ quantità queste di solito molto piccole, risulta opportuno prendere qualche volta come valore maggiorante di $H\left(t_{r+1}-t_{1}\right)$ l'espressione:

$$
\left\{\frac{\partial(w)}{\omega}\right\}_{\max }+\frac{\left\{\frac{\partial^{2} \omega}{\partial J^{2}}\right\}_{\max }\left(M_{a} \Delta_{m}\left(t+M_{b} \Delta_{m} b\right)+\left\{\frac{\partial^{2} \omega}{\partial J \partial \alpha}\right\}_{\max } \Delta_{m} a+\left\{\frac{\partial^{2} \omega}{\partial J \partial b}\right\}_{\max } \Delta_{m} b\right.}{\omega_{\min }}
$$

dove il simbolo max o min accanto alle parentesi indica il massimo o il minimo del valore assoluto della espressione entro parentesi per ogni $J$ compreso fra $y_{\max }+\eta, y_{\min }-\eta$ e ogni $t$ di $\left(0, t^{\prime}\right)$. Analoghe considerazioni si possono fare per $K_{a} S\left(t_{r+1}-t_{r}\right)$.

(1) Cioè per un valore di $J(t)$ in questo intervallo.

(2) Si noti che $\mathrm{i}$ valori di $t$ in cui sono calcolati $a(t)$ e $b(t)$ possono anche essere diversi. 
Si può calcolare facilmente un altro limite superiore per $H\left(t_{\gamma^{*}+1}-t_{1}\right)$, $K_{a}\left(t_{v+1}-t_{r}\right)$ qualora sia $\omega=f(J) e^{h a+k b}$ con $h$ e $k$ numeri indipendenti da $J, a, b$. Si ha infatti

$$
\begin{gathered}
t_{r+1}-t_{r}=\frac{e^{-h a\left(t_{r}\right)-k b\left(t_{r}\right)}}{f\left(J\left(t_{r}\right)\right)} \\
H=\left|f^{\prime}(J(\bar{t})) e^{h a(t)+k b(t)}\right|
\end{gathered}
$$

dove $t$ e $\bar{t}$ sono valori intermedi fra $t_{r}$ e $t_{*^{\prime}+1}$. Quindi:

$$
H\left(t_{r+1}-t_{r}\right) \leq\left\{\begin{array}{l}
\left\{f^{\prime}(J(t))\right\}_{\max } \\
\{f(J(t))\}_{\min }
\end{array} e^{|h| \Delta_{m} a+|k| \Delta_{m} b}\right.
$$

Si ha poi usando le stesse notazioni :

$$
K_{a}=\left|h f\left(J^{\prime}\right) e^{h a(t)+\cdot h b(t)}\right|
$$

Per cui :

$$
K_{a}\left(t_{r+1}-t_{v}\right)<|h| \frac{\{f(J)\}_{\max }}{\{f(J)\}_{\min }} e^{|h| \Delta_{n i} a+|k| \Delta_{m b} b}
$$

dove $|f(J)|_{\max }\{f(J)\}_{\min }\left\{f^{\prime}(J)\right\}_{\min }$ significano rispettivamente il massimo o il minimo di $|f(J)|\left|f^{\prime}(J)\right|$ per $J$ variabile fra $y_{\max }+\eta, y_{\min }-\eta\left({ }^{1}\right)$.

Se occorre un limite superiore più basso si può serivere:

$$
\begin{gathered}
\left|f\left(J^{\prime}\right)\right| \leq\left|f\left(J\left(t_{v}\right)\right)\right|+\left|J^{\prime}-J\left(t_{,}\right)\right|\left\{f^{\prime}(J)\right\}_{\max } \leq \\
\quad \leq f\left(J\left(t_{,}\right)\right)+\left\{f^{\prime}(J)\right\}_{\max }\left(M_{a} \Delta_{m} a+M_{b} \Delta_{m} b\right)
\end{gathered}
$$

e una forma analoga vale per $f^{\prime}(J)$. Allora si ha:

$$
\begin{aligned}
& H\left(t_{r+1}-t,\right)=\left(\left\{\frac{f^{\prime}(J)}{f(J)}\right\}_{\max }+\frac{\left\{f^{\prime \prime}(J)\right\}_{\max }}{\{f(J)\}_{\min }}\left(M_{a} \Delta_{m} a+M_{b} \Delta_{m} b\right)\right) e^{|h| \Delta_{m} a+|k| \Delta_{m} b} \\
& K_{a}\left(t_{r+1}-t_{n}\right) \leq|h|\left(1+\frac{\left\{f^{\prime}(J)\right\}_{\max }}{\{f(J)\}_{\min }}\left(M_{a} \Delta_{m} a+M_{b} \Delta_{m} b\right)\right) e^{|h| \Delta_{m} a+|z| \Delta_{m} b}
\end{aligned}
$$

col solito significato per i termini entro parentesi graffa. Analoghe considerazioni valgono per $K_{b}\left(t_{r+1}-t_{r}\right)$.

Passiamo ora allo studio di $\Delta_{m} a$ e $\Delta_{m} a^{\prime}$. Quando queste grandezze non sono assegnate come dato del problema e non sono facilmente calcolabili in base alle formule che le esprimono conviene calcolare di esse un limite superiore, in base alle seguenti considerazioni.

(1) Si noti che se $h$ è positivo e $a$ decrescente si può sopprimere nell' esponenziale il $\Delta_{m} a$. Lo stesso accade se $h$ è negativo e $a$ crescente. Le stesse conclusioni valgono con le ovvie modificazioni per il parametro $b$. 
Cominciando da $\Delta_{m} \alpha$ si deve notare che esso è sempre inferiore ad $\frac{\alpha_{\max }^{\prime}}{\omega_{\min }}$ essendo $a_{\max }^{\prime}$ il valore massimo $d \mathbf{i}\left|a^{\prime}\right|$ in $\left(0, t^{\prime}\right)$, $\omega_{\min }$ il valore minimo di $\omega$ in $\left(0, t^{\prime}\right)$ e per $J$ compreso fra $y_{\max }+\eta, y_{\min }-\eta$. Di questo limite superiore abbiamo fatto varie applicazioni nelle altre nostre note relative agli invarianti adiabatici.

Però si può trovare per $\Delta_{m} \alpha$ un limite superiore che di regola è più basso di quello ora accennato. Infatti se $\bar{t}$ è un punto dell'intervallo $\left(t_{r}, t_{r+1}\right)$ abbiamo:

$$
a^{\prime}(t)=a^{\prime}\left(t_{r}\right)+\left(t-t_{r}\right) a^{\prime \prime}(\bar{t})
$$

Allora si ha:

$$
\begin{aligned}
& \int_{t_{r}}^{t_{r+1}}\left|a^{\prime}\right| d t \leq\left|a^{\prime}\left(t_{v}\right)\right| \int_{t_{r}}^{t_{r+1}} d t+\left|\int_{t_{r}}^{t_{r+1}} a^{\prime \prime}(\bar{t})\left(t-t_{r}\right)\right| \\
\leq & \left|\frac{a^{\prime}\left(t_{r}\right)}{\omega\left(J\left(t_{r}\right), a\left(t_{r}\right), b\left(t_{r}\right)\right)}\right|+\frac{\left|a^{\prime \prime}\right| \max }{2}\left(t_{r+1}-t_{r}\right)^{\prime}
\end{aligned}
$$

dove $\left|a^{\prime \prime}\right|_{\max }$ è il valore massimo di $\left|a^{\prime \prime}\right|$ in $\left(0, t^{\prime}\right)$. Quindi si ha subito:

$$
\Delta_{n t} a \leq\left|\frac{a^{\prime}}{\omega}\right|_{\max }+\frac{\left|\alpha^{\prime \prime}\right|_{\max }}{2 \omega^{\prime \prime} \min }
$$

indicando con $\left|\frac{\alpha^{\prime}}{\omega}\right|_{\max }$ il valore massimo di $\left|\frac{\alpha^{\prime}}{\omega}\right|$ per ogni $t$ di $\left(0, t^{\prime}\right)$ e per ogni $J$ compreso fra $y_{\max }+\eta, y_{\min }-\eta$.

Ora siccome avviene spesso in pratica che $\frac{\left|\alpha^{\prime \prime}\right|_{\max }}{2 \omega^{2}{ }_{\min }}$ è traseurabile rispetto $\left|\frac{\alpha^{\prime}}{\omega}\right|_{\max }$ e questọ termine è inferiore dal più uguale ad $\frac{a_{\max }^{\prime}}{\omega_{\min }}$ se ne conclude che questo limite superiore per $\Delta_{m} a$ può essere più conveniente del. l'altro calcolato con la $\frac{a_{\max }^{\prime}}{\omega_{\min }}$. È da notare che ponendo fin da principio nelle nostre formule in luogo $\left|a^{\prime}\right|$ il suo limite superiore $\left|a^{\prime}\right|_{\max }$ il termine $S\left(M_{a} H+K_{a}\right)\left(t_{r+1}-t_{r}\right)$ nell' espressione di $Z_{r}$ va moltiplicato per $\frac{1}{3}$ mentre il termine analogo nell'espressione di $Z_{r}^{\prime}$ va diviso per 2 , e $V\left(\alpha, t^{\prime}\right)$ viene sostituito con $\left|\alpha^{\prime}\right|_{\max } t^{\prime}$.

Passiamo ora allo studio di $\Delta_{m} a^{\prime}$. Un suo limite superiore è evidente. mente $\frac{\left|a^{\prime \prime}\right|_{\max }}{\omega_{\min } a_{\min }^{\prime}}$ essendo $a_{\min }^{\prime}$ il valore minimo di $\left|a^{\prime}\right|$ in $\left(0, t^{\prime}\right)$. Oppure si 
può moltiplicare denominatore e numeratore dell'espressione di $\Delta_{t_{r}} a^{\prime}$ per $t_{r+1}-t_{r}$ e allora un limite superiore di $\Delta_{m} \alpha^{\prime}$ è dato da $\frac{\left|\alpha^{\prime \prime}\right|_{\max }}{\omega_{\min } \Delta_{\min } a}$ essendo $\Delta_{\min } a$ il valore minimo di $\Delta_{t_{r}} a$ in $\left(0, t^{\prime}\right)$.

Questi limiti superiori hanno però l'inconveniente di non essere applicabili qualora in qualche punto di $\left(0, t^{\prime}\right)$ sia $a^{\prime}$ o $\Delta_{t} a$ nulla. Conviene nei casi ora ricordati modificare un po' la forma del termine $M_{a} \Delta_{m} \alpha^{\prime} V(a, t)$. Sí ricordi perciò che questo termine sorge dalla somma di integrali del tipo:

Ora si ha subito la relazione:

$$
\left|\int_{t_{r}}^{t_{y+1}} \alpha^{\prime \prime} \int_{t_{r}}^{t} J_{a}^{\prime} d t\right|
$$

$$
\left|\int_{t_{r}}^{t_{r+1} t} a^{\prime \prime} \int_{t_{r}}^{t} J_{a}^{\prime} d t\right| \leq M_{a}\left|a^{\prime \prime}\right|_{\max } \int_{t_{r}}^{t_{r+1}} d t \int_{t_{r}}^{t} d t=\frac{M_{a}\left|a^{\prime \prime}\right|{ }_{\max }\left(t_{r+1}-t_{, r}\right)}{2 \omega_{\min }} .
$$

Per cui nelle espressione di $I(t)$ in luogo di $M_{a} V\left(a, t^{\prime}\right) \Delta_{t} a^{\prime}$ si può mettere $\frac{M_{a}\left|a^{\prime \prime}\right|_{\max } t^{\prime}}{2 \omega_{\min }}$. Questa sostituzione è stata sempre fatta nelle nostre note sugli invarianti adiabatici.

Oppure si può osservare che è:

$$
\left|\int_{t_{r}}^{t_{r}+1} a^{\prime \prime} \int_{t_{r}}^{t} J_{a} d t\right|<\frac{M_{a}}{\omega_{\min }} \int_{t_{r}}^{t_{r+1}}\left|a^{\prime \prime}\right| d t
$$

per cui in luogo $M_{a} V\left(\alpha, t^{\prime}\right) \Delta_{m} a^{\prime}$ possiamo mettere (s' intende nella espressione di $\left.I\left(t^{\prime}\right)\right) \frac{M_{a}}{\omega_{\min }} V\left(a^{\prime}, t^{\prime}\right)$.

E poi ovvio che in qualche caso speciale dalle formule di definizioni di $\Delta_{t} a, \Delta_{t} \alpha^{\prime}$ si possono ottenere valori per $\Delta_{m} a, \Delta_{m} \alpha^{\prime}$ sfruttando le particolari proprietà di $a$ e $a^{\prime}\left({ }^{1}\right)$.

4. Caso in cui $b$ è costante. Calcolo di $t^{\prime}$ e altre proprietà. - Rimandando al Capitolo terzo lo studio del caso in cui $b$ è variabile, supponiamo d'ora in poi $b$ costante per cui $y(t)$ si riduce a $J_{0}$ (valore di $J$ per $t=0$ ) e

(1) Osserviamo che in seguito indicheremo i massimi o minimi di $\left|a^{\prime}\right| 0\left|a^{\prime \prime}\right|$ sempli* cemente con i simboli $a_{\max }^{\prime}$ o $a_{\max }^{\prime \prime}$. 
resta così :

$$
\left|J-J_{0}\right| \leq\left|\int_{0}^{t} a^{\prime} J_{a} d t\right| \leq\left(Z \Delta_{m} a+M_{a} \Delta_{m} a^{\prime}\right) V\left(a, t^{\prime}\right)+M_{a} \Delta_{m} a=I\left(t^{\prime}\right) .
$$

Da questa formula troviamo un metodo per verificare se $t^{\prime}$ è compreso entro l'intervallo $(0, h)$. Per questo scopo osserviamo anzitutto che $I\left(t^{\prime}\right)$ è definita in tutto $\left(0, h^{\prime}\right)$ perchè per tutti i valori $t^{\prime}$ di questo intervallo si possono definire $Z, M_{a}, \Delta_{n} a, \Delta_{m} a^{\prime}, V\left(a, t^{\prime}\right)$. Osserviamo poi che $I\left(t^{\prime}\right)$ è una funzione non decrescente di $t^{\prime}$ perchè è prodotto e somma di termini che sono tali. Naturalmente questa $I\left(t^{\prime}\right)$ è limite superiore di $\left|J-J_{0}\right|$ nell' intervallo $(0, h)$ in cui $J_{0}-\eta<J<J_{0}+\eta$.

Ora è facile dimostrare il teorema: se $I\left(t^{\prime}\right)$ è per $t^{\prime}<h^{\prime}$ inferiore a $\eta$ certamente $t^{\prime}$ è interno a $(0, h)$ e lo stesso accade di tutti i $\bar{t}^{\prime}$ inferiori a $t^{\prime}$.

Infatti o l'intervallo $(0, h)$ coincide con $\left(0, h^{\prime}\right)$ e allora il nostro teorema è dimostrato, oppure $h<h^{\prime}$ allora $\left|J(h)-J_{0}\right|=\eta$. Ma se $I\left(t^{\prime}\right)<\eta$ non può essere $t^{\prime}>h$ altrimenti si avrebbe $I\left(t^{\prime}\right) \geq I(h) \geq \eta$ contro l'ipotesi. Perciò la verifica se $t^{\prime}$ è interno ad $h$ e quindi vale la (47) si ottiene provando che $I\left(t^{\prime}\right)$ è inferiore a $\eta$.

$\grave{\mathrm{E}}$ interessante il caso in cui si possa prendere $\eta$ abbastauza piccolo per cui sia possibile $h^{\prime}=\infty$. Allora se $I\left(t^{\prime}\right)$ resta per $t \rightarrow \infty$ inferiore a $\eta$ la $I\left(t^{\prime}\right)$ si può applicare in tutto l'intervallo $(0, \infty)$ cịoè la $(47)$ può servire per prevedere valori assintotici di $\left|J-J_{0}\right|$. 主 da notare che dovendo restare $I\left(t^{\prime}\right)$ finito occorre che $V\left(\alpha, t^{\prime}\right)$ resti pure finito $\left({ }^{1}\right)$.

Si noti ora che se $\Delta_{m} a$ e $\Delta_{m} \alpha^{\prime}$ si possono considerare infinitesime cioè se $a$ varia in maniera infinitamente lenta e graduale, allora se per ogni $t^{\prime}$ finito o infinito la $V\left(a, t^{\prime}\right)$ è finita resulta $\left|J-J_{0}\right|$ inferiore per ogni $t$ finito a qualunque $\varepsilon$ minore di $\eta_{i}$ e percio per l'arbitrarietà di $\varepsilon, J=J_{0}$ per ogni valore di $t$ quindi anche al limite per $t \rightarrow \infty$. Si ha così che se un parametro varia in maniera infinitamente lenta e graduale la $J$ resta costante per tutto l'intervallo anche infinito in cui $V\left(\alpha, t^{\prime}\right)$ resta finita purchè esista un numero $\eta$ tale che per ogni $t^{\prime}$ sia $J_{2}-\eta>J_{0}>J_{1}+\eta$. Sotto questa forma viene spesso enunciato il concetto d'invariante adiabatico $\left({ }^{2}\right)$.

(1) In seguito nella espressione di $I(t)$ porremo talvolta in lnogo di $M_{a}$ soltanto $M$.

(z) Notiamo che $i$ fisiei sostitnisenno spesso alla nozione di variazione graduale del parametro quella di variazione nón in fase col moto. Questa nozione è più generale ma più. difficile a mettere in equazione. 
5. Applicazione al problema del moto armonico con forza di richiamo dipendente dal tempo. - Intenderemo per moto armonico con forza di ri. chiamo dipendente dal tempo, un moto retto dall' equazione:

$$
\frac{d^{2} q}{d t^{2}}=-\omega^{2} q
$$

essendo $q$ la coordinata e $\omega$ una funzione reale e positiva del tempo. Evidentemente questo moto per $\omega$ costante è periodico, e ciò per ogni $\omega>0$ e per ogni $p, q$, quindi $C$ coincide con $(0, \infty)$, e il corrispondente $A$ con tutto il piano. Come abbiamo visto in altri lavori ( $\left.{ }^{1}\right)$ ponendo:

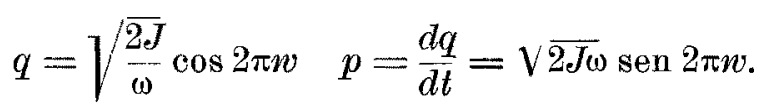

Abbiamo subito:

$$
\mathscr{H}=\frac{1}{2} p^{2}+\frac{\omega^{2} q^{2}}{2}=\omega J
$$

il che ci dice $\mathscr{H}$ funzione univoca di $J$ e $\frac{\partial \mathscr{H}}{\partial J}>0$. Di più poichè $\mathscr{H}$ può as. sumere qualunque valore positivo la $J$ varia da zero a infinito per cui $J_{1}=0, J_{2}=\infty$ cioè $J_{1}$ e $J_{2}$ variano con continuità e il campo $J, \omega$ è con. vesso. In base alle (49) abbiamo subito con trasformazioni esposte nella nostra nota citata $\left({ }^{2}\right)$ :

$$
\begin{aligned}
& \frac{d J}{d t}=-J a^{\prime} \operatorname{sen} 4 \pi w \\
& \frac{d w}{d t}=\frac{e^{a}}{2 \pi}+\frac{a^{\prime}}{4 \pi} \cos 4 \pi w
\end{aligned}
$$

essendo $a=\log \omega . \dot{\mathrm{E}}$ facile vedere che $\omega=e^{a}, J_{a}=\operatorname{sen} 4 \pi w, w_{a}=\cos 4 \pi \omega$ sono funzione limitate e Lischiptziane per ogni $a$ di $(-\infty, \infty), J$ di $(0, \infty)$, $w$ di $(-\infty, \infty)$ verificando perciò che sono soddisfatte tutte le condizioni necessarie per applicare le nostre formule.

Ora nella equazione (50) si può prendere come incognita non $J$ ma $\log J$ ottenendosi la formula:

$$
\frac{d \log J}{d t}=-\alpha^{\prime} \operatorname{sen} 4 \pi x
$$

Poichè $J$ può variare fra i limiti $(0, \infty) \log J$ può variare fra $(-\infty,+\infty)$ perciò qualunque sia il valore iniziale di $J, J_{0}$ e qualunque sia $\eta$ (finito)

(i) Cfr. il primo lavoro citato a pag. 1.

(2) Si osservi che la $v$ vale la $\theta$ della nota citata divisa per $2 \pi$. 
l'intervallo $\left(0, h^{\prime}\right)$ coincide con $(0, \infty)$ in quanto chè per qualunque $\eta$ finito $\log J_{0} \pm \eta$ è sempre entro i limiti in cui le soluzioni di (48) sono periodiche. Ora dalla (52) si ha subito $M_{a}=1, R=P=0, S=4 \pi$ mentre dalla (51) resulta $H=0, K_{a}=\frac{e^{a}}{2 \pi}, A=\frac{1}{4 \pi}$. Quanto a $\left(t_{r+1}-t_{r}\right)$ si può prendere $\pi e^{-a\left(t_{r}\right)}$ perchè le $J_{a}$ e $w_{a}$ sono periodiche di periodo $\frac{1}{2}$ rispetto a $w$. Allora si ha per la (39) e tenendo presente questo valore di $t_{r+1}-t_{r}, K_{a}\left(t_{r+1}-t_{r}\right)=\frac{e^{\Delta_{m}} a}{2}$. Quindi si ha:

$$
Z=\pi e^{\Delta_{m} \alpha}+\frac{1}{2}
$$

Allora per le nostre formule si ha:

$$
\left|\log \frac{J\left(t^{\prime}\right)}{J_{0}}\right| \leq\left(\pi e^{\Delta_{m} a}+\frac{1}{2}\right) \Delta_{m} a V\left(a, t^{\prime}\right)+\Delta_{m} a^{\prime} V\left(a, t^{\prime}\right)+\Delta_{m} a=I\left(t^{\prime}\right) .
$$

E questa equazione vale per qualunque $t^{\prime}$ tale che $V\left(a, t^{\prime}\right)$ sia finita. Infatti la nostra relazione vale per qualunque $t^{\prime}<h$ con $h$ scelta in modo che in $(0, h)$ sia:

$$
\left|\log \frac{J\left(t^{\prime}\right)}{J_{0}}\right|<\eta
$$

Ora per provare se $t^{\prime}$ è in $h$ basta provare che $I\left(t^{\prime}\right)$ è inferiore a $\eta$. Ma $\eta$ non appare nell' espressione di $I\left(t^{\prime}\right)$ perciò possiamo prenderla a nostro arbitrio purchè finita e quindi la sceglieremo sempre superiore ad ogni $I\left(t^{\prime}\right)$ finito ('). Resta così provato la nostra asserzione e cioè si può applicare la (54) per qualunque $t^{\prime}$ ehe rende $V\left(a, t^{\prime}\right)$ finito.

Ora nella nostra nota del 1932 avremo trovato la formula (scambiando in essa $t_{1}$ con $t^{\prime}$ )

$$
\log \frac{J}{J_{0}}=\frac{\pi\left|\omega^{\prime}\right|_{\max }}{\omega_{\min }^{2}}+\left(\pi+\frac{\pi^{2}}{3}\right) \frac{\left|\omega_{\max }^{\prime}\right|^{2}}{\omega_{\min }^{4}} \omega_{\max } t^{\prime}+\frac{\pi \omega_{\max }^{\prime \prime}}{2} \omega_{\max } t^{\prime}
$$

dove il simbolo max o min posto al fianco di una espressione indica il valore massimo o minimo di quella espressione in valore assoluto.

Per fare un raffronto fra il secondo membro di (55) e quello di (54) è bene modificare la $\left(5^{\prime}\right)$ per avere termini fra loro confrontabili sebbene sia probabile che la (54) dia sempre resultati migliori della (55). Per fare questo

(i) Più precisamente fissato un $I\left(t^{\prime}\right)$ finito sceglieremo $\eta$ superiore a $I\left(t^{\prime}\right)$. Con ciò resta provato che $t^{\prime}$ e in $(O, h)$. 
confronto basta dunque mettere in luogo del termine $\Delta \alpha^{\prime \prime} V(a, t)$ il termine $\frac{\alpha_{\text {max }}^{\prime \prime}\left(t_{r^{*}+1}-t_{\eta^{\prime}}\right)}{2}$ come si è già indicato nel paragrafo 4 . Di pì̀ si ha:

$$
\begin{aligned}
& \Delta_{m a} a<\alpha_{\max }^{\prime}\left(t_{r+1}-t_{,}\right)_{\max }=\pi \frac{\omega_{\max }^{\prime}}{\omega_{\text {min }}^{2}} \quad \frac{\omega_{\max }}{\omega_{\min }}>1, \\
& V\left(\alpha, t^{\prime}\right)=\int_{0}^{t^{\prime}}\left|\frac{d \omega}{d t}\right| d \omega \leq\left|\frac{\omega^{\prime}}{\omega}\right|_{\max } t^{\prime} \leq \frac{\omega_{\max }^{\prime}}{\omega_{\min }} t^{\prime} \\
& \frac{a_{\text {max }}^{\prime \prime}\left(t_{r+1}-t_{r}\right)_{\max } t^{\prime}}{2} \leq \frac{\pi \omega^{\prime \prime}{ }_{\text {max }}}{2} t^{2}{ }_{\min }^{\prime}+\frac{\pi \omega^{\prime 2}{ }_{\max }}{2} t^{3}{ }_{\min }^{\prime} .
\end{aligned}
$$

Ponendo poi sin da principio in luogo di $\left|\alpha^{\prime}\right|$ il suo valore massimo $\alpha_{\max }^{\prime}$ la $Z$ diventa $\frac{\pi}{3} e^{\Delta_{m z} a}+\frac{1}{2}$. Essendo poi di solito $\Delta_{m} a$ molto piccolo si può ammettere $\frac{\pi^{2}}{3} e^{\Delta_{m} a}+\pi\left({ }^{1}\right)$ sia poco diverso da $\frac{\pi^{2}}{3}+\pi$.

Ora un semplice raffronto fra la (54) e (55) tenendo conto delle (56) e (57) ei fa vedore che le formule ora ottenute danno in generale limitazioni più basse di quelle di noi trovate qualche anno fa.

6. Applicazione al problema delle masse rariabili. - In base alle formule sovrascritte potremo calcolare con molta facilità un limite superiore per le variazioni dell' eccentricità dell'orbita osculatrice nel problema dei due corpi di massa variabile. Otteremo delle limitazioni non molto diverse da quelle trovate per il caso delle masse decrescenti nella nostra nota del 1934 già citata, ma le troveremo molto più rapidamente e valide qualunque sia il modo di variazione della massa.

In base al resultato delle nostre note (2) il problema dei due corpi di massa variabile si riconduce al seguente sistema Hamiltoniano in cui $c$ è la costante delle aree $B$ il prodotto della massa totale per la costante di gravitazione $K$ :

$$
\frac{d p}{d t}=-\frac{B}{r^{2}}+\frac{c^{2}}{r^{3}}, \quad \frac{d r}{d t}=p, \quad \mathscr{H}=\frac{p^{2}}{2}-\frac{2 B}{r}+\frac{c^{2}}{2 r^{2}} .
$$

Ora ̀̀ notissimo che per qualunque valore di $B$ positivo esiste un campo $C$ in cui le soluzioni del sistema (58) sono periodiche. Perciò in questo campo si potrà costruire la $J$ e la corrispondente eccentricità $\varepsilon$ che vale

(1) Se $a$ è crescente allora per l'osservazione di pag. 15 si può porre in questa for mula $\Delta_{i z} a=0$.

(2) Vedi in particolare la citata nota dell" Aceademia di Torino"* 
$\sqrt{1-\frac{4 \pi^{2} c^{2}}{J^{2}}}$. 亡̀ poi noto che le $(58)$ hanno soluzioni periodiche fino a quando $\varepsilon$ è inferiore a 1 e positivo, perciò $\mathrm{i}$ valori $J_{1}, J_{2}$ saranno $\mathrm{i}$ valori $4 \pi c$ e $\infty$ corrispondenti a $\varepsilon=0, \varepsilon=1$.

Perciò fino a che le $p$ e $q$ restano nella $C$ ossia fino a che $\varepsilon$ resta compreso fra 0 e 1 si può trasformare le equazioni (58) in altre contenenti la $J$ e la sua variabile coniugata $w$ e in base all'osservazione contenuta alla fine del paragrafo secondo possiamo ricondurci a equazioni che hanno per incognite $\varepsilon$ e $w$. Queste equazioni già ottenute in altre note sono della forma:

$$
\begin{gathered}
\frac{d \varepsilon}{d t}=-a^{\prime} \frac{\left(1-\varepsilon^{2}\right) \cos u}{1-\varepsilon \cos u} \\
\frac{d w}{d t}=\frac{e^{2 a}\left(1-\varepsilon^{2}\right)^{\frac{3}{2}}}{2 \pi c^{3}}+\frac{a^{\prime}}{2 \pi}\left(\frac{\operatorname{sen} u}{\varepsilon}+\frac{\left(1 \cdot \varepsilon^{2}\right) \operatorname{sen} u \cos u}{1-\varepsilon \cos u}\right) .
\end{gathered}
$$

In cui $a=\log B, u$ è una grandezza definita dalla equazione di KePLERo

$$
2 \pi w=u-\varepsilon \operatorname{sen} u \text {. }
$$

Evidentemente nel caso delle masse costanti $u$ è l'anomalia eccentrica e $w$ l'anomalia media. Le (59), (60) valgono dunque per $0<\varepsilon<1$.

Noi dovremo supporre naturalmente le condizioni iniziali tali che $\varepsilon_{0}$ resulti compreso fra 0 e 1 .

Dalle formule sovra scritte è poi facile vedere che $H=-\frac{2 \pi B^{2}}{J^{2}}$ e quindi $\frac{\partial \omega}{\partial J}>0$ e che $J_{a}$ e $w_{a}$ soddisfano alle condizioni poste per esse. Noi porremo poi sempre $\eta<1-\varepsilon_{0}, \eta<\varepsilon_{0}$ in modo che l'intervallo $\left(0, h^{\prime}\right)$ in cui $\varepsilon_{0}+\eta<\varepsilon_{2}, \varepsilon_{0}-\eta>\varepsilon_{1}$ coincida con l'intervallo $(0, \infty)$.

Posto ciò passiamo al calcolo di $I\left(t^{\prime}\right)$. Abbiamo intanto i seguenti limiti superiori per (') $M_{a}, R, S, P$, validi per qualunque $w$ e $t$ e per $\varepsilon$ compreso fra $\varepsilon_{\max }=\varepsilon_{0}+\eta, \varepsilon_{\min }=\varepsilon_{0}-\eta\left({ }^{2}\right)$ :

$$
\begin{gathered}
M_{b}=\left(1+\varepsilon_{\max }\right), \quad R=\left\{\frac{\partial J_{a}}{\partial \varepsilon}\right\}=\left\{\frac{1+2 \varepsilon \cos u}{1-\varepsilon \cos u}-\frac{\left(1-\varepsilon^{2}\right) \cos ^{2} u}{(1-\varepsilon \cos u)^{2}}+\frac{\partial J_{a}}{\partial u} \frac{\partial u}{\partial \varepsilon}\right\} \\
=\left\{\frac{2 \varepsilon \cos u}{1-\varepsilon \cos u}-\frac{\left(1-\varepsilon^{2}\right) \cos ^{2} u}{(1-\varepsilon \cos u)^{2}}+\left(\frac{\left(1-\varepsilon^{2}\right) \operatorname{sen} u}{1-\varepsilon \cos u}+\frac{\left(1-\varepsilon^{2}\right) \varepsilon \operatorname{sen} u \cos u}{(1-\varepsilon \cos u)^{2}}\right) \frac{\partial u}{\partial \varepsilon}\right\} .
\end{gathered}
$$

(1) Qui intendiamo per $J_{a}$ e $w_{a}$ i termini che moltiplicano $a^{\prime}$ nelle (59). Naturalmente anche le $J_{a}$ e $w_{a}$ soddisferebbero a quelle condizioni.

(2) La parentisi graffa attorno ad alcuni termini significa che questi termini sono calcolati in modulo per un valore di $a(t)$ e nno di $\varepsilon(t)$ con $t$ intermedio rra $\left(t_{*}, t_{r+1}\right)$ in modo da soddisfare a quelle condizioni per cui si introducono nelle nostre formule. 
Ma è:

$$
2 \pi w=u-\varepsilon \operatorname{sen} u \text {. }
$$

Quindi derivando rispetto a $\varepsilon$

$$
\frac{\partial u}{\partial \varepsilon}=\frac{\operatorname{sen} u}{1-\varepsilon \cos u} .
$$

Perciò si ha:

$$
R=\left\{\frac{2 \varepsilon \cos u}{1-\varepsilon \cos u}-\frac{\left(1-\varepsilon^{2}\right) \cos ^{2} u}{(1-\varepsilon \cos u)^{2}}+\frac{\left(1-\varepsilon^{2}\right) \operatorname{sen}^{2} u}{(1-\varepsilon \cos u)^{3}}\right\} \leq \frac{1+3 \varepsilon_{\max }}{1-\varepsilon_{\max }}+\frac{1+\varepsilon_{\max }}{\left(1-\varepsilon_{\max }\right)^{2}} .
$$

Si ha poi:

$$
\begin{gathered}
S=\left\{\frac{\partial J_{a}}{\partial u} \frac{\partial u}{\partial w}\right\}=\left\{\frac{\left(1-\varepsilon^{2}\right) \operatorname{sen}^{2} u}{(1-\varepsilon \cos u)^{3}} \cdot 2 \pi\right\} \leq \frac{2 \pi\left(1+\varepsilon_{\max }\right)}{\left(1-\varepsilon_{\max }\right)^{2}} \\
A=\frac{1}{2 \pi}\left\{\frac{\operatorname{sen} u}{\varepsilon}+\frac{\left(1-\varepsilon^{2}\right) \operatorname{sen} u \cos u}{1-\varepsilon \cos u}\right\} \leq \frac{1}{2 \pi}\left(\frac{1}{\varepsilon_{\min }}+1+\varepsilon_{\max }\right) \\
P=0 .
\end{gathered}
$$

Passiamo ora al calcolo di $H\left(t_{r+1}-t_{r}\right)$ e $K_{a}\left(t_{r+1}-t_{r}\right)$. Essendo $\omega$ del tipo $f(\varepsilon) e^{h a}$ con $f(\varepsilon)=\frac{\left(1-\varepsilon^{2}\right)^{\frac{3}{2}}}{2 \pi c^{3}}$ e $h=2$ si ha dalla (41), dopo semplici considerazioni :

e dalla (42)

$$
H\left(t_{r+1}-t, r\right) \leq\left(\frac{+3 \varepsilon_{\max } e^{2 \Delta_{m} a}}{\left(1-\varepsilon_{\max }^{2}\right)}+\frac{3\left(1-2 \varepsilon_{\min }^{2}\right)\left(1+\varepsilon_{\max }\right)}{\left(1-\varepsilon_{\max }^{2}\right)^{2}} \Delta_{m} a e^{2 \Delta_{m} a}\right)
$$

$$
K_{a}\left(t_{r+1}-t_{.}\right)=\left(2 e^{2 \Delta_{m} a}+\frac{6 \varepsilon_{\max }\left(1+\varepsilon_{\max }\right)\left(1-\varepsilon^{2}{ }_{\min }\right)^{\frac{1}{2}}}{\left(1-\varepsilon_{\max }\right)^{\frac{3}{2}}} \Delta_{m} a e^{2 \Delta_{m} a}\right) .
$$

Si ha allora:

$$
\begin{aligned}
& Z=\frac{1}{2}\left\{\frac{\left(1+3 \varepsilon_{\max }\right)\left(1+\varepsilon_{\max }\right)}{1-\varepsilon_{\max }}+\frac{\left(1+\varepsilon_{\max }\right)^{2}}{\left(1-\varepsilon_{\max }\right)^{2}}\right\}+ \\
& +\frac{2 \pi \cdot 3 \varepsilon_{\max }}{\left(1-\varepsilon_{\max }\right)^{2}} \frac{\left(1+\varepsilon_{\max }\right)^{2}}{\left(1-\varepsilon_{\max }^{2}\right)} e^{2 \Delta_{m} a}+2 \pi \frac{2\left(1+\varepsilon_{\max }\right)}{\left(1-\varepsilon_{\max }\right)^{2}} e^{2 \Delta_{m} a} \\
& \frac{\left(1+-\varepsilon_{\max }\right)}{\left(1-\varepsilon_{\max }\right)^{2}}\left(\frac{1}{\varepsilon_{\min }}+\left(1+\varepsilon_{\max }\right)\right)+ \\
& +\frac{2 \pi\left(1+\varepsilon_{\max }\right)}{\left(1-\varepsilon_{\max }\right)^{2}} \Delta_{n} a e^{2 \Delta_{m} a}\left\{\frac{3\left(1-2 \varepsilon_{\min }^{2}\right)\left(1+\varepsilon_{\max }\right)^{2}}{\left(1-\varepsilon_{\max }^{2}\right)^{2}}+\frac{6 \varepsilon_{\max }\left(1+\varepsilon_{\max }\right)\left(1-\varepsilon_{\min }^{2}\right)^{\frac{1}{2}}}{\left(1-\varepsilon_{\max }^{2}\right)^{\frac{3}{2}}}\right\} \text {. }
\end{aligned}
$$

Ora è da notare che a una formula non molto diversa siamo giunti nel nostro lavoro citato a pag. 2 e precisamente questa $Z$ (supponendo nullo $\Delta_{m} a$ ) a un valore minore della $\theta$ che in tali note abbiamo calcolato. Anzi essendo 
$\Delta_{m} a$ nelle applicazioni sempre molto piccolo possiamo ritenere la $\theta$ sempre superiore a $Z$ perció faremo le deduzioni seguenti in base ai valori numerici di $\theta$ che abbiamo già calcolato in altra occasione.

Abbiamo dunque applicando la (32)

$$
\left|\varepsilon-\varepsilon_{0}\right| \leq \theta V\left(a, t^{\prime}\right) \Delta_{m} a+\left(1+\varepsilon_{\max }\right) \Delta_{m} a^{\prime} V\left(a, t^{\prime}\right)+\left(1+\varepsilon_{\max }\right) \Delta_{m} a
$$

- otteniamo così una formula analoga a quella trovata nelle nostre note precedenti ma valida per qualunque modo di variazione della massa. E questa formula si può applicare per tutto l'intervallo $(0, h)$ in cui la $\left|\varepsilon-\varepsilon_{0}\right|$ resta inferiore a $\eta$. Anzi da essa è facile dedurre un limite inferiore per la varia zione di massa necessaria affinchè $\left|\varepsilon-\varepsilon_{0}\right|$ raggịnga il valore prefissato $\eta$. Difatti si ha che quel valore di $t^{\prime}$ che rende il secondo membro di (62) uguale a $\eta$ è certamente inferiore $o$ al più uguale al valore $h$ in cui $\varepsilon-\varepsilon_{0}$ paò valere $\eta$. Siccome $V\left(a, t^{\prime}\right)$ è crescente se ne deduce che il valore di $V\left(a, t^{\prime}\right)$ che soddisfa il secondo membro di $(62)$ eguagliato a $\eta$ dà il cercato limite inferiore. Supponendo ora la massa $m(t)$ sempre erescente o decrescente, si ha $V\left(a, t^{\prime}\right)=\left|\log \frac{m\left(t^{\prime}\right)}{m_{0}}\right|$ (essendo $m_{0}$ la somma delle masse dei due corpi per $t=0$ ) e perciò:

$$
\left|\log \frac{m\left(t^{\prime}\right)}{m_{0}}\right|=\frac{\eta-\left(1+-\varepsilon_{\max }\right) \Delta_{m} a}{\theta \Delta_{m} a+\left(1+\varepsilon_{\max }\right) \Delta_{m} a^{\prime}}(')
$$

Vediamo ora di applicare queste formule al caso delle stelle doppie in cui la massa diminuisce per irraggiamento, caso gia da noi studiato nelle note citate. In esse supponendo $\eta=\frac{1}{100}$ si è trovato $\theta$ inferiore a 200. Ovviamente poi $1+\varepsilon_{\max }<2$. Resta da conoseere il valore di $\Delta_{m} a$ e $\Delta_{m} a^{\prime}$. Ora per il sistema Terra-Sole all'epoca presente questo numero secondo il JEANS sarebbe dell'ordine di $10^{-13}$. In altre epoche questo numero poteva essere diverso perchè tale era la massa del sole. Però si può pensare che non sia variato di molto al trascorrere del tempo perchè se è ovvio che più grande è la massa più grande è l'energia irradiata, questo éffetto può essere, almeno in parte, compensato dal fatto che più grande è la massa più breve resulta di solito il periodo. Comunque poichè le stelle doppie hanno masse e periodi diversi dal sistema sole-terra noi faremo i nostri calcoli pex diversi valori di $\Delta_{m} \alpha$.

Quanto a $\Delta_{m} a^{\prime}$ i dati sono molto più incerti, noi faremo per ciò l'ipotesi

(1) S'intende che $\gamma_{i}$ si suppone superiore a $\left(1+\varepsilon_{m a x}\right) \Lambda_{m} a^{\prime}$ 
(in qualche caso particolare verificata (')) che esso sia dell'ordine di grandezza di $\Delta_{m} a$. Allora avremo dalla (63) ponendo per semplificare i calcoli e per stare più al sicuro $\Delta_{m} \alpha^{\prime}=100 \Delta_{m} a$

$$
\left|\log \frac{m\left(t^{\prime}\right)}{m_{0}}\right|=\frac{\frac{1}{100}-2 \Delta_{m} a}{400 \Delta_{n} a}
$$

e ponendo $\Delta_{m} \alpha=10^{-n}$ e ricordando che in quesio caso la massa è decrescente

$$
\left.m_{n} m^{\prime}\right)=e^{0,25\left(10-2-2 \cdot 10^{-n}\right) 10^{n-2}} .
$$

Si vede subito da questa formula che $n=13$ si ha sensibilmente $\frac{m_{0}}{m(t)}=e^{2,5.10^{8}}$ cioè per avere variazioni $d \mathrm{i} \varepsilon$ di un centesimo la massa dovrebbe subire una enorme diminuzione. Dalla (64) si vede subito che il valore $\frac{m_{u}}{m}$ pur diminuendo $m$ rimane enorme fino a che $n \geq 6$. Esso può diventare possibile solo per valori di $n$ minori di 6 . Si può perciò concludere che per avere variazione anche piccolissime di eccentricità occorre invocare nelle stelle doppie enormi variazioni di masse, o valori per le variazioni relative di massa in un periodo troppo diversi da quelli comunemente ammessi.

Agli stessi resultati eravamo giunti in altre note considerando però particolari leggi di variazioni delle masse. Qui i resultati sono ottenuti in modo molto piủ generale, e con calcoli più facili. Vi è però l'ipotesi di $\Delta a^{\prime}$ dell'ordine di $\Delta a$ ipotesi che non sappiamo controllare in generale essendo del resto tale controllo di competenza degli astronomi. Comunque ci sembra di aver trovato delle formule per lo studio delle variazioni di eccentricità al variare della massa ancora più semplici di quelle da noi date nelle altre note.

Vogliamo da ultimo osservare che $V(\alpha, t)=\log \frac{m_{0}}{m(t)}$ se la massa ̀̀ de-

(1) Si noti che nel caso della legge del JeAss, (Astronomy and Cosmogony; pag. 130-131) si ha $\frac{d B}{d t}=-\propto B^{3}$ con $\propto$ costante e la nostra ipotesi su $\Delta a^{\prime}$ risulta verificata. Difatti $d a=-\alpha e^{z a}, \Delta a=\alpha \int_{t_{r}}^{t_{r+1}} e^{z a} d t, \Delta \alpha^{\prime}=2 x \int_{t_{r}}^{t_{r+1}} e^{4 \pi-2 a} d t$ dove $\bar{a}$ ̀े un valore di $t$ intermedio fra $t_{r}$ e $t_{r+1}$. Siccome $a$ varia in $t_{r}, t_{r+1}$ di quantità trascurabili si può suporre $e^{2(a-a)}=1 \mathrm{e}$ percio resulta subito $\Delta a^{\prime}=2 \Delta a$ cioè $\Delta a$ e $\Delta a^{\prime}$ sono dello stesso ordine di grandezza. Si noti che il ragionamento vale ponendo in luogo dell'esponente $\mathbf{3}$ qualunque esponente e perciò $\dot{\theta}$ applicabile anche per la legge proposta dall'Armellini ( Rendiconti Lincei » II sem., 1932). 
crescente, tende all'infinito per $t \rightarrow \infty$ se $m(t)$ per $t \rightarrow \infty$ tende allo zero. In questo caso le formule ora sviluppate non ei permettono di affermare che $\mid \varepsilon-\varepsilon, j$ resta sempre limitato perchè il secondo membro di (62) tende all'infinito. Ciò è conforme del resto a resultati dell'ArmellonI ( ${ }^{1}$ ) che ha mostrato la $\varepsilon$ tendere per $t \rightarrow \infty$ all'infinito qualora $m(t)$ tenda allo zero (sempre per $t \rightarrow \infty$ ) d'ordine sufficientemente grande. Quindi nel caso ora ricordato $i$ nostri metodi non ci permettono di prevedere valori assintotici per $\varepsilon$ al tendere di $t$ all'infinito.

Però se $m(t)$ per $t \rightarrow \infty$ non tende allo zero, allora se $a(t)$ è tale che $V(a, t)$ resti sempre finita (come accade spesso in questioni che si presentano nella pratica) le nostre formule conservano sempre valori finiti e da esse si potrà, almeno in qualche occasione, prevedere l'andamento assintotico di $\varepsilon$. Ritroviamo così in un caso particolare quanto si è affermato in generale e cioè che le formule sviluppate in questo lavoro, permettono a differenza di quelle usate nelle nostre note precedenti, di studiare variazioni di grandezze per intervalli di lunghezza infinita.

\section{CAPITOLO II.}

\section{Considerazioni generali sull'integrazione approssimata di equazioni} differenziali col metodo degli invarianti adiabatici. - Nella nostra nota del 1932 già citata abbiamo integrato approssimativamente, usando il concetto d'invariante adiabatico, l'equazione del moto armonico con forza variabile col tempo, determinando anche un limite superiore per l'errore che si commette sostituendo quella soluzione approssimata alla soluzione esatta.

Si presenta perciò spontanea l'idea di estendere tale metodo d'integrazione approssimata a qualunque sistema meccanico con un grado di libertà del tipo ora considerato con un solo parametro $a$ variabile.

Questa estensione non ci è riuscita, anzi non la riteniamo possibile. Per ginstificare questa asserzione crediamo opportuno ripetere la dimostrazione più naturale per il calcolo della soluzione approssimata e del limite superiore per l' errore commesso sostituendo la soluzione approssimata a quella esatta, e vedremo che detto limite superiore non resta affatto piccolo in tutto l'in. tervallo in cui $J$ si può ritẹnere costante come avviene nel caso sovra ricordato del moto armonico.

(1) " Rendiconti Accademia dè Lincei », II sem., 1982. 
Si consideri perciò il nostro sistema (5) in cui si porrà $b^{\prime}=0$ essendo ora soltanto a variabile. Avremo allora:

$$
\left\{\begin{array}{l}
\frac{d J}{d t}=-a^{\prime} J_{a} \\
\frac{d w}{d t}=\omega+a^{\prime} w_{a}
\end{array}\right.
$$

e integrando da $\left(0\right.$, a $\left.t^{\prime}\right)$ queste equazioni abbiamo:

$$
\begin{gathered}
J=J_{0}-\int_{0}^{t^{\prime}} a^{\prime} J_{a} d t \\
v=w_{0}+\int_{0}^{t^{\prime}} \omega d t+\int_{0}^{t^{\prime}} a^{\prime} w_{a} d t .
\end{gathered}
$$

Ora si è visto che l'integrale a secondo membro di (66) un certo intervallo $\left(0, t^{\prime}\right)$ equivale ad una quantità finita moltiplicata per $\Delta_{m} \alpha$ o $\Delta_{m} \alpha^{\prime}$. Perciò se $\Delta_{m} a, \Delta_{m} a^{\prime}$ sono abbastanza piccole si può ritenere in $\left(0, t^{\prime}\right), J=J_{0}$

Passiamo alla (67). Per l'ultimo integrale che in essa vi figura si po. trebbero applicare i metodi già usati per l'integrale a secondo membro di (18) metodi però che sarebbero validi nel caso in cui fosse $\int_{w}^{w+1} w_{a} d t=0$ es. sendo l'integrazione fatta supponendo $a$ e $J$ costanti. Questa ipotesi non è necessariamente sempre verificata nella pratica ma essa può presentarsi in vari casi particolari per esempio nel problema dei due corpi di massa variabile. Allora ammessa tale ipotesi l'ultimo integrale a secondo membro di (67) resulta dell' ordine di $\Delta_{m} a$ o $\Delta_{m} a^{\prime}$ e quindi trascurabile se tali sono $\Delta_{m} a, \Delta_{m} a^{\prime}$. Ma a secondo membro di (65) si presenta l'integrale di $\omega$ il quale non si può calcolare in modo esatto perchè $\omega$ è in generale funzione di $J$ e si sa soltanto che la $J$ vale approssimativamente $J_{0}$ nell' intervallo $\left(0, t^{\prime}\right)$.

A prima vista si potrebbe pensare che il sostituire nella espressione di $\omega$ in luogo di $J, J_{0}$ (ottenendo così una soluzione approssimata di (65)) conduca ad errori piccoli, ma calcolando per la via più naturale il limite superiore dell' errore commesso sostituendo nella (65) $J_{0}$ a $J$ esso non risulta dell'ordine di $\Delta_{m} a$. Infatti indicato con $\omega(J, a), \omega\left(J_{0}, \alpha\right)$ rispettivamente il valore vero di $\omega$ e quello che si ottiene quando in luogo di $J$ si pone $J_{0}$ l'errore $\mu$ che si commette sostituendo nell' integrale di $\omega, J_{0}$ in luogo di $J$ : sarebbe

$$
\mu=\left|\int_{0}^{t}\left(\omega(J, a)-\omega\left(J_{0}, a\right)\right) d t\right| \text {. }
$$


Per trovare un limite superiore di questo errore, si potrebbe procedere così ricordando che $\left|\omega(J, a)-\omega\left(J_{0}, \alpha\right)\right| \leq H\left|J-J_{0}\right|\left({ }^{1}\right)$

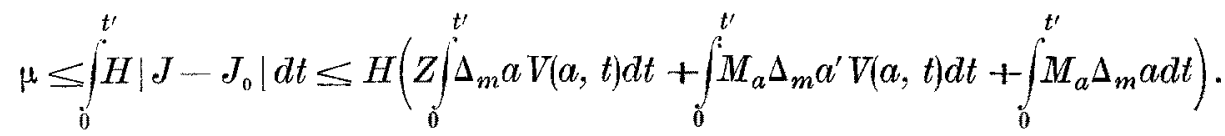

Il limite superiore per $\mu$ che ora si calcola non è dell'ordine di $\Delta_{m} a$. Per semplificare la dimostrazione supponiamo a variabile in modo lineare ciò̀ $\Delta \alpha^{\prime}=0, \Delta_{m} a=\frac{\alpha^{\prime}}{\omega_{\min }}, V(\alpha, t)=\alpha^{\prime} t$. Si ha allora:

$$
\mu \leq \frac{H Z}{\omega_{\min }} \int_{0}^{t^{\prime}} a^{\prime z} t d t+H \int_{0}^{t^{\prime}} \frac{M_{a}}{\omega_{\min }} a^{\prime} d t=\frac{H Z}{2 \omega_{\min }}\left(a^{\prime} t^{\prime}\right)^{2}+\frac{H M_{a}}{\omega_{\min }} a^{\prime} t^{\prime}
$$

e siccome all'altimo membro di $(70)$ non compare, $\Delta_{m} a$ ma $\left(\alpha^{\prime} t^{\prime}\right)^{2}$ ossia $\left(V\left(a, t^{\prime}\right)\right)^{2}$ quantità questa che si suppone finita $\left(^{2}\right)$ e perciò il limite superiore ora calcolato non è affatto trascurabile. Per questa ragione risulta dubbia e forse non lecita la sostituzione della $J_{0}$ a $J$ nella (67).

Si obietterà che un limite superiore dell' errore commesso calcolato per altra via potrebbe resultare dell'ordine di $\Delta_{m} a$. Non mi è riuscito di dimostrare che questo fatto sia, almeno in generale, da escludersi, e del resto una dimostrazione del genere non avrebbe un eccessivo interesse. Vogliamo però notare che le considerazioni sopra riportate per il calcolo del limite superiore per $\mu$ si possono facilmente ripetere soltanto supponendo $\left|J-J_{0}\right|$ dell'ordine di $\Delta_{m} a V\left(a, t^{\prime}\right)$ e $\frac{\partial \omega}{\partial J}$ sempre dello stesso segno per $t$ in $\left(0, t^{\prime}\right)$ e $J$ compreso fra $J_{0}-\eta, J_{0}+\eta$ e si troverebbe che $\mu$ è dell'ordine di $\left(V\left(a, t^{\prime}\right)\right)^{2}$. Ciò ci fa appunto ritenere che la soluzione approssimata ora costruita non sia valida in tutto l'intervallo $\left(0, t^{\prime}\right)$ in cui $J$ si può ritenere uguale a $J_{0}$.

Nel caso del moto armonico tale difficoltà non si presenta, perchè on non dipende da $J$ e perciò nel secondo integrale di (67) non è necessario sostitrire $J$ a $J_{0}$.

(1) La $H$ si suppone relativo a tutto l'intervallo $\left(0, t^{\prime}\right)$, ossia ora $H$ è il valore massimo di $\frac{\partial w}{\partial J}$ per $J$ cómpreso fra $J_{0}-\eta, J_{0}+\eta$ e $t$ in $\left(0, t^{\prime}\right)$.

${ }^{(2)}$ A rigore in questa equazione compare anche un termine in $a^{\prime} t^{\prime}$. Ma come vedremo questo termine si può fare almeno in qualche caso scomparire. Kesta però il fatto che $\mu$ è inferiore solo a un termine in $V\left(a, t^{\prime}\right)$. 
8. Integrazione approssimata di equazioni differenziali col metodo degli invarianti adiabatici. - Nonostante le affermazioni di carattere negativo del numero precedente dalle formule $(66),(67)$ si può ancora ottenere una inte. grazione approssimata delle (65) purchè si limiti convenientemente l'intervallo $\left(0, t^{\prime}\right)$ in cui l'integrazione approssimata sia sostituibile a quella esatta, e si ammetta qualche altra ipotesi spesso verificata in pratica.

Noi supporremo anzitutto che $\frac{\partial V}{\partial a}$ e quindi $w_{a}$ siano funzioni periodiche di $w$ a valore medio nullo nell' intervallo $w, w+1$ e ciò per qualunque $w$, per qualunque $J$ compreso fra $J_{0}+\eta, J_{0}-\eta$ e per qualunque $a(t)$. S'intende che il valor medio di $\frac{\partial V}{\partial \alpha}$ si suppone calcolato supponendo $J$ e $a$ costante e solo variabile $w$.

In secondo luogo ammetteremo che nell' intervallo $\left(^{1}\right)\left(0, \frac{1}{\omega(J(0), a(0))}\right)$ esista un valore $t_{v}$ di $t$ tale che in esso sia $\frac{\partial V}{\partial a}\left(J\left(t_{0}\right), \alpha\left(t_{0}\right), w\left(t_{0}\right)\right)=0$. Questa ipotesi può ritenersi verificata in pratica per le seguenti ragioni. Si consideri la funzione $\frac{\partial V^{\prime}}{\partial a}=\frac{\partial V}{\partial a}(J(0), a(0), w(0)+\omega(0) t)$ questa funzione essendo in $\left(0, \frac{1}{\omega(0)}\right)$ periodica a valor medio nullo assumerà in questo intervallo valori in parte positivi ed in parte negativi. Perciò se per fissare le idee la $\frac{\partial V^{\prime}}{\partial a}$ è in $t=0$ positiva esisterà certamente un punto $\bar{t}$ dell'intervallo $\left(0, \frac{1}{\omega(0)}\right)$ in cui questa funzione è negativa. Ora poichè, come si è già visto varie volte e avremo occasione di ricordare più innanzi, la differenza fra $J(t)-J(0), a(t)-a(0)$, $w(t)-\left(w(0)+\frac{t}{\omega(0)}\right)$ è in tutto $\left(0, \frac{1}{\omega(0)}\right)$ dell'ordine di $\Delta_{m} a$ in pratica molto piccolo possiamo ritenere per continuità in $\vec{t} \frac{\partial V}{\partial a}$ negativa e percio esisterà in $(0, \bar{t})$ ossia in $0, \frac{1}{\omega(0)}$ un valore $t_{0}$ per cui $\frac{\partial V}{\partial a}$ è nulla. Del resto con le considerazioni che precedono è facile verificare l'esistenza di $t_{n}$.

Ciò posto torniamo alle nostre formule è osserviamo che si avrebbe:

$$
w=w(0)+\int_{0}^{t^{\prime}} \omega(J, a) d t+\int_{0}^{t^{\prime}} a^{\prime} w_{a} d t .
$$

(i) Per brevitá V'estremo superiore di questo intervallo verrà indicato eon $\frac{1}{\omega(0)}$. 
Noi prenderemo come valore approssimato di $w$ l'espressione $w$ tale che (1):

$$
\bar{w}=w(0)+\int_{0}^{t^{\prime}} \omega\left(J\left(t_{0}\right), a\right) d t+\int_{0}^{t^{\prime}} a^{\prime} w_{a} d t .
$$

Sottraendo membro a membro la (71) dalla (72) si ha:

$$
|w-\bar{w}| \leq\left|\int_{0}^{t^{\prime}} \omega(J, a) d t-\omega\left(J\left(t_{0}\right), a\right) d t\right|+\left|\int_{0}^{t^{\prime}} a^{\prime} w_{a} d t\right| .
$$

Quindi trovando un limite superiore del secondo membro di questa equazione ne segue subito un limite superiore per il primo. Ora ricordando la proprietà di $w_{a}$ e ripetendo gli stessi calcoli fatti per il calcolo del limite

(1) Sỉ noti che si ammette di conoscere $J\left(t_{0}\right)$ invece di $J_{0}$. $\mathrm{E}$ possibile però calcolare un valure approssimato di $J\left(t_{0}\right), J_{1}\left(t_{0}\right)$ tale che la sua introduzione in (72) porti ad un errore dell' ordine di $t_{m} a \cdot a_{\text {max }}^{\prime} t^{\prime}$ per le nostre approssimazione traseurabile. Noi porremo:

$$
J_{1}\left(t_{0}\right)=J_{0}-\int_{0}^{t_{0}} a^{\prime} J_{a}^{\prime} d t
$$

Essendo $J_{a}^{\prime}$, analogamente al capitolo primo $J_{a}$, calcolata per $J=J_{0}, a=a(0)$, $\omega=\omega(0)+\frac{t}{\omega(0)}$. Allora integrando la (12) sottraendovi la $(\alpha)$ e ragionando nel modo con cui si è giunti alla (24) sỉ ha:

Quindi:

$$
J\left(t_{0}\right)-J_{1}\left(t_{0}\right) \mid \leq Z\left(\Delta_{m} a\right)^{2} \leq \frac{Z}{\omega_{\min }} \Delta_{m} a \cdot a^{\prime}
$$

$$
\left|\int_{0}^{t^{\prime}}\left(\omega\left(J\left(t_{0}\right), a\right)-\omega\left(J_{1}\left(t_{0}\right), a\right)\right) d t\right| \leq \frac{Z H}{\omega \min } \Delta_{m} a \cdot a_{\max }^{\prime} t^{\prime}
$$

come dovevasi dimostrare.

Naturalmente si ammette nota anche $t_{0}$. Questo valore si potrebbe calcolare approssimativamente nel seguente modo. Si indichi con $\left(\frac{\partial V^{\prime}}{\partial a}\right)$ la $\frac{\partial V}{\partial a}\left(J_{0}, a(0), w(0) t\right)$, questa differisee come si è detto da $\frac{\partial V}{\partial a}$ di un termine dell' ordine di $\Delta a$, termine il cui massimo indicheremo con $P \Delta_{m a} a$ essendo $P$ un numero facilmente calcolabile. Allora $t_{0}$ sarà compreso fra quel valore $t^{\prime}$ in cui $\frac{\partial V^{\prime}}{\partial a}-P \Delta_{m} a>0$ e quel valore $t^{\prime \prime}$ in cui $\frac{\partial V}{\partial a}+P \Delta_{m} a<0$. Ammetteremo l' esi. stenza di $t^{\prime}, t^{\prime \prime}$ e che $t^{\prime \prime}-t^{\prime}$ sia dell' ordine di $\Delta_{n} a$. Allora ragionando come precede è facile provare che se si prende come valore approssimato di $t_{0}$ un qualunque valore fra $t^{\prime}$ e $t^{\prime \prime}$ si commette ancora un errore dell'ordine di $\Delta_{m} a \cdot a^{\prime} \max t^{\prime}$ cioè un errore trascurabile. Si potrebbe cercare anche un metodo di approssimazioni successive per il calcolo di $t_{0}$ che evi. tasse le ipotesi fatte in questa nota, ma non ne vale la pena giacchè questa ipotesi sono assai spesso verificate in pratica e del resto in pratica $J\left(t_{0}\right)$ e $t_{0}$ si ha nei dati del problema stesso. 
superiore per $\left|J-J_{0}\right|$ si può dire che è:

$$
\left|\int_{0}^{t^{\prime}} a^{\prime} w_{a} d t\right|=\left(\bar{Z} \Delta_{m} a+A \Delta_{m} a^{\prime}\right) V\left(a, t^{\prime}\right)+A \Delta_{m} a
$$

essendo la $\bar{Z}$ costruita in modo analogo alla $Z$ che appare nel limite superiore di $\left|J-J_{0}\right|$. Resta da trovare un limite superiore per il primo termine a secondo membro di (73).

A questo scopo si osservi che è valida la relazione:

$$
\left|\int_{0}^{t^{\prime}} \omega(J, a)-\int_{0}^{t^{\prime}} \omega\left(J\left(t_{0}\right), a\right) d t\right| \leq\left|\int_{0}^{t_{0}}\left(\omega(J, a)-\omega\left(J\left(t_{0}\right), a\right)\right) d t\right|+\left|\int_{t_{0}}^{t^{\prime}}\left(\omega(J, a)-\omega\left(J\left(t_{0}\right), a\right)\right) d t\right| .
$$

Ora è :

$$
\left|\int_{0}^{t_{0}} \omega(J, a)-\omega\left(J\left(t_{0}\right), a\right) d t\right| \leq H \int_{0}^{t_{0}}\left|J-J\left(t_{0}\right)\right| d t \leq \frac{M H \Delta_{m} a}{\omega(0)}
$$

Molto più difficile è il calcolo di un valore maggiorante per l'ultimo integrale a secondo membro di (75). A questo scopo dividiamo l'intervallo $\left(t_{0}, t^{\prime}\right)$ in $n$ parti con i punti $\tau_{1}, \tau_{2}, \ldots \tau_{n-1}$ punti tali che:

$$
\tau_{1}=t_{0}+\frac{1}{\omega\left(J\left(t_{0}\right), a\left(t_{0}\right)\right)} \quad \tau_{r+1}=\tau_{r}+\frac{1}{\omega\left(J\left(\tau_{r}\right), a\left(\tau_{r}\right)\right)} .
$$

Potremo perciò serivere:

$$
\begin{aligned}
& \left|\int_{t_{0}}^{t^{\prime}}\left(\omega(J, a)-\omega\left(J\left(t_{0}\right), a\right)\right) d t\right| \leq\left|\int_{t_{0}}^{\tau_{1}}\left(\omega(J, a)-\omega\left(J\left(t_{0}\right), a\right)\right) d t\right|+ \\
& +\left|\int_{\tau_{r}}^{\tau_{r+1}}\left(\omega(J, a)-\omega\left(J\left(t_{0}\right), a\right)\right) d t\right| \ldots \cdot\left|\int_{t_{n-1}}^{t}\left(\omega(J, a)-\omega\left(J\left(t_{0}\right), a\right)\right) d t\right| .
\end{aligned}
$$

Consideriamo ora l'integrale generico:

$$
\begin{aligned}
\left|\int_{\tau_{r}}^{\tau_{r+1}} \omega(J, a)-\omega\left(J\left(t_{0}\right), a\right) d t\right| \leq\left|\int_{\tau_{r}}^{\tau_{r+1}}\left(\omega(J, a)-\omega\left(J\left(\tau_{r}\right), a\right)\right) d t\right|+ \\
+\left|\int_{\tau_{r}}^{\tau_{r+1}}\left(\omega\left(J\left(\tau_{r}\right), a\right)-\omega\left(J\left(t_{0}\right), a\right)\right) d t\right| .
\end{aligned}
$$

(1) La relazione $\left|J\left(t_{0}\right)-J\right| \leq \frac{M \Delta_{m} a}{\omega(0)}$ valida per $t$ in $\left(0, t_{0}\right)$ si ottiene subito dalla prima di (65) dopo averla integrata da $t$ a $t_{0}$. 
Rifacendo lo stesso calcolo già fatto a proposito della espressione per il limite superiore di $J\left(t_{r}\right)-J_{0}$

$$
\begin{gathered}
\left|J\left(\tau_{r}\right)-J\left(t_{0}\right)\right| \leq\left(Z \Delta_{m} a+M \Delta_{m} a^{\prime}\right) V\left(\alpha, \tau_{r}\right)\left({ }^{1}\right) \\
\left|J\left(\tau_{r}\right)-J\left(t_{0}\right)\right| \leq \frac{Z}{\omega_{\min }} a^{\prime z}{ }_{\max } \tau_{r}+\frac{M}{2} \frac{a_{\max }^{\prime \prime}}{\omega_{\min }} \tau_{r}
\end{gathered}
$$

con il noto significato per $a_{\max }^{\prime}, \alpha^{\prime \prime}{ }_{\max }$.

Perciò si ha subito ricordando $t^{\prime}>\tau_{r}$

$$
\begin{aligned}
& \left|\int_{\tau_{r}}^{\tau_{r+1}} \omega\left(J\left(\tau_{r}\right), a\right)-\omega\left(J\left(t_{0}\right), a\right) d t\right| \leq H \int_{\tau_{r}}^{\tau_{r+1}}\left|J\left(\tau_{r}\right)-J_{0}\right| d t \leq \\
& \leq H\left(\frac{Z a^{\prime 2}{ }_{\max }}{\omega_{\min }}+\frac{M}{2} \frac{a_{\text {max }}^{\prime \prime}}{\omega_{\min }}\right) t^{\prime}\left(\tau_{r+1}-\tau_{r}\right) .
\end{aligned}
$$

Quanto all'altro termine a secondo membro di (79) sono necessarie varie osservazioni. Anzitutto si noti che si ha:

$$
\begin{aligned}
& \text { (82) } \omega(J, a)=\omega\left(J\left(\tau_{r}\right), a\left(\tau_{r}\right)\right)+\left(\frac{\partial \omega}{\partial J}\right)_{\tau_{r}}\left(J(t)-J\left(\tau_{r}\right)\right)+\left(\frac{\partial \omega}{\partial J}\right)_{\tau_{r}}\left(a(t)-a\left(\tau_{r}\right)\right)+ \\
& +\left\{\frac{\partial^{*} \omega}{\partial J^{2}}\right\}\left(J(t)-J\left(\tau_{r}\right)\right)^{2}+2\left\{\frac{\partial^{2} \omega}{\partial J \partial a}\right\}\left(J(t)-J\left(\tau_{r}\right)\right)\left(a(t)-a\left(\tau_{r}\right)\right)+\left\{\frac{\partial^{2} \omega}{\partial a^{2}}\right\}\left(a(t)-a\left(\tau_{r}\right)\right)^{2} .
\end{aligned}
$$

Dove $\left(\frac{\partial \omega}{\partial J}\right)_{\tau_{r}},\left(\frac{\partial \omega}{\partial a}\right)_{\tau_{r}}$ significa il valore di queste derivate calcolate per $J=J\left(\tau_{r}\right)$, $a=a\left(\tau_{r}\right)$ mentre le derivate entro parentesi graffa significano valori di queste derivate per valori di $J(\tau)$ e $a(\tau)$ per $\tau$ in $\tau_{r}, \tau_{r+1}$.

Con analogo significato dei simboli si ha:

$$
\omega\left(J\left(\tau_{r}\right), a\right)=\omega\left(J\left(\tau_{r}\right), a\left(\tau_{r}\right)\right)+\left(\frac{\partial \omega}{\partial a}\right)_{\tau_{r}}\left(a(t)-a\left(\tau_{r}\right)\right)+\left\{\frac{\partial^{2} \omega}{\partial J^{2}}\right\}^{2}\left(a(t)-a\left(\tau_{r}\right)\right)^{2} .
$$

Allora se col suffisso max posto vicino ad una certa grandezza intendiamo al solito il massimo in valore assoluto di questa grandezza per $J$ compreso fra $J_{0}-\eta$ e $J_{0}+\eta$ e $a$ compresa fra $\left(0, t^{\prime}\right)$. Abbiamo sottraendo la (83) dalla (82) e sostituendo nel secondo membro di (79):

$$
\left|\int_{\tau_{r}}^{\tau_{r+1}} \omega(J, a)-\omega\left(J\left(\tau_{r}\right), a\right) d t\right| \leq\left|\left(\frac{\partial \omega}{\partial J}\right)_{\tau_{r}}\right|\left|\int_{\tau_{r}}^{\tau_{r+1}}\left(J(t)-J\left(\tau_{r}\right)\right) d t\right|+L \alpha_{\max }^{\prime 2}\left(\tau_{r+1}-\tau_{r}\right)^{3}
$$

(1) A rigore in lnogo di $V\left(a, \tau_{r}\right)$ si dovrebbe mettere la variazione totale di $a$ in $t_{0}, \tau_{\gamma^{*}}$ Ma essendo questa variazione inferioxe a $V\left(\alpha, \tau_{r}\right)$ conviene mettere nellà (80) la $V\left(\alpha, \tau_{r}\right)$. 
con

$$
\left.L=\frac{1}{3}\left\{\frac{\partial^{2} \omega}{\partial J^{2}}\right\}_{\max } M_{a}^{2}+\frac{2}{3}\left\{\frac{\partial^{2} \omega}{\partial J \partial \alpha}\right\}_{\max } M_{a}+\frac{2}{3}\left\{\frac{\partial^{2} \omega}{\partial \alpha^{2}}\right\}_{\max }{ }^{1}\right)
$$

Calcoliamo ora un limite superiore per $\left|\int_{\tau_{r}}^{\tau_{r+1}}\left(J(t)-J\left(\tau_{r}\right)\right) d t\right|$. Si ricordi anzitutto:

$$
\frac{d J}{d t}=-a^{\prime} J_{a}=-a^{\prime} \frac{\partial^{2} V}{\partial a \partial w}
$$

Si osservi poi che è :

$$
\begin{gathered}
\left|\frac{\partial^{2} V(J(t), w(t), a(t))}{\partial a \partial w}-\frac{\partial^{2} V\left(J\left(\tau_{r}\right), w\left(\tau_{r}\right)+\frac{t}{\tau_{r+1}}-\tau_{r}, a\left(\tau_{r}\right)\right)}{\partial a \partial w}\right| \leq \\
\leq R\left|J(t)-J\left(\tau_{r}\right)\right|+S\left|w(t)-w\left(\tau_{r}\right)-\frac{t-\tau_{r}}{\tau_{r+1}-\tau_{r}}\right|+P\left|a(t)-a\left(\tau_{r}\right)\right| .
\end{gathered}
$$

E ricordando che $v\left(\tau_{r}\right)+\frac{t-\tau_{r}}{\tau_{r+1}-\tau_{r}}$ è analoga alla $w_{1}$ usata nel paragrafo 2 del primo eapitolo si può affermare che in base alla (21) il termine che moltiplica $S$ è inferiore a $\left(M_{a} H+K_{a}\right)\left(\tau_{r+1}-\tau_{r}\right)+A \int_{\tau_{r}}^{t}\left|\alpha^{\prime}\right| d t$.

Si ha così indicando per brevità con $\left|\frac{\partial V(t)}{\partial a \partial w}-\begin{array}{c}\partial V\left(\tau_{r}\right) \\ \partial a \partial w\end{array}\right|$ il primo membro di (87)

$$
\begin{aligned}
\left|\frac{\partial V(t)}{\partial a \partial w}-\frac{\partial V\left(\tau_{r}\right)}{\partial \alpha \partial w}\right| & \leq\left(R M_{a}+S\left(M_{a} H+K_{a}\right)\left(\tau_{r+1}-\tau_{r}\right)+S A+P\right) \int_{\tau_{r}}^{t}\left|\alpha^{\prime}\right| d t \\
& \leq 2 Z a_{\max }^{\prime}\left(t-\tau_{r}\right) .
\end{aligned}
$$

Quindi indicato per maggiore chiarezza $\left\{\frac{\partial V}{\partial a \partial w}\right\}_{\tau_{r}}$ e $\left\{\frac{\partial V}{\partial a}\right\}_{\tau_{r}}$ rispettivamente (*) la $\frac{\partial^{2} V}{\partial a \partial w}, \frac{\partial V}{\partial a}$ calcolate per $J=J\left(\tau_{r}\right), w=w\left(\tau_{r}\right)+\frac{t-\tau_{r}}{\tau_{r+1}-\tau_{r}}, a=a\left(\tau_{r}\right)$ e con $a^{\prime \prime}$ un

(1) Ciò si ottiene ricordando che $J(t)-J\left(\tau_{r}\right)<M_{a} a_{\max }^{\prime}\left(t-\tau_{\gamma}\right),\left|\alpha(t)-a\left(\tau_{r}\right)\right| \leq a_{\max }^{\prime}\left(t-\tau_{r}\right)$.

(2) Si noti che $\left(\frac{\partial^{2} V}{\partial a_{\partial} w}\right)_{\tau_{r}}$ coincide con $\frac{\partial V\left(\tau_{v}\right)}{\partial a \partial v}$ sopra considerata. 
valore di $a^{\prime \prime}$ per $t$ compreso fra $\tau_{r}$ e $\tau_{r+1}$ si ha subito dalla (86):

$$
\begin{gathered}
J(t)-J\left(\tau_{r}\right)=\int_{\tau_{r}}^{t} a^{\prime} \frac{\partial^{2} V}{\partial a \partial w} d t=a^{\prime}\left(\tau_{r}\right) \int_{\tau_{r}}^{t} \frac{\partial^{2} V}{\partial a \partial w} d t+\int_{\tau_{r}}^{t} a_{M}^{\prime \prime}\left(t-\tau_{r}\right) \frac{\partial^{2} V}{\partial a \partial w} d t= \\
=a^{\prime}\left(\tau_{r}\right) \int_{\tau_{r}}^{t}\left\{\begin{array}{l}
\partial^{2} V \frac{V}{\partial} a w \\
\tau_{\tau_{r}}
\end{array} d t+g(t)=\frac{a^{\prime}\left(\tau_{r}\right)}{\omega_{r}}\left[\left\{\frac{\partial V}{\partial a}\right\}\right]_{\tau_{r}}^{t}+g(t)\right.
\end{gathered}
$$

dove si è indicato con $g(t)$ l'espressione:

$$
g(t)=\int_{\tau_{r}}^{t} \frac{\partial^{2} V}{\partial a \partial w} a_{M}^{\prime \prime}\left(t-\tau_{r}\right) d t+a^{\prime}\left(\tau_{,}\right) \int_{\tau_{r}}^{t}\left(\frac{\partial^{2} V}{\partial a \partial w}-\left(\frac{\partial^{2} V}{\partial a \partial w}\right)_{\tau_{r}}\right) d t
$$

e con $\omega_{r}$ la $\omega\left(J\left(\tau_{r}\right), \alpha\left(\tau_{r}\right)\right)$. Abbiamo subito:

$$
|g(t)| \leq-\frac{M_{a} a^{\prime \prime}{ }_{\max }\left(t-\tau_{r}\right)^{2}}{2}+Z a_{\text {max }}^{\prime 2}\left(t-\tau_{r}\right)^{2} .
$$

Si ha così :

$$
\begin{aligned}
& \left|\int_{\tau_{r}}^{\tau_{r}+1}\left(J(t)-J\left(\tau_{r}\right)\right) d t \leq\right| \frac{a^{\prime}\left(\tau_{r}\right)}{\omega_{r}} \int_{\tau_{\tau_{r}}}^{\tau_{r}+t}\left(\frac{\partial V}{\partial a}\right)_{\tau_{r}} d t-\frac{a^{\prime}\left(\tau_{r}\right)}{\omega_{r}{ }^{2}}\left|\frac{\partial V\left(J\left(\tau_{r}\right), w\left(\tau_{r}\right), a\left(\tau_{r}\right)\right)}{\partial a}\right|+ \\
& +\left|\int_{\tau_{r}}^{\tau_{r+1}} g(t) d t\right|=+\left|\frac{a^{\prime}\left(\tau_{r}\right)}{\omega_{r}^{2}} \frac{\partial V}{\partial a}\left(J\left(\tau_{r}\right), w\left(\tau_{r}\right), a\left(\tau_{r}\right)\right)\right|+\left|\int_{\tau_{r}}^{\tau_{r+1}} g(t) d t\right| .
\end{aligned}
$$

Il primo termine a secondo membro si è potuto omettere in base alla relazione :

$$
\int_{\tau_{r}}^{\tau_{r}+1}\left\{\frac{\partial V}{\partial a}\right\}_{\tau_{r}} d t=\frac{1}{\omega_{r}} \int_{w\left(\tau_{r}\right)}^{\partial v\left(\tau_{r}\right)+1} \frac{\partial V\left(J\left(\tau_{r}\right), w\left(\tau_{r}\right)+\frac{t-\tau_{r}}{\tau_{r+1}-\tau_{r}}, \alpha\left(\tau_{r}\right)\right.}{\partial a} d w
$$

e all'ipotesi che il valor medio di $\frac{\partial V}{\partial a}$ calcolato da $w$ a $w+1$ con $J$ e $a$ costante è nullo.

Allora per trovare un limite superiore per $\left|\int_{\mathfrak{x}_{r}}^{\tau}\left(J(t)-J\left(\tau_{r}\right)\right)\right| d t$ rimane da trovarsi un limite superiore per $\frac{\partial V}{\partial a}$ calcolato per $t=\tau_{r}$ valore che ora indicheremo con $\frac{\partial V}{\partial a}\left(\tau_{r}\right)$. 
Ora possiamo scrivere: ricordando che $\frac{\partial V}{\partial a}\left(t_{0}\right)=0$

$$
\left|\begin{array}{c}
\partial V\left(\tau_{r}\right) \\
\partial a
\end{array}\right| \leq\left|\frac{\partial V\left(\tau_{\tau}\right)}{\partial a}-\frac{\partial V\left(\tau_{r-1}\right)}{\partial a}\right| \ldots\left|\frac{\partial V\left(\tau_{s}\right)}{\partial a}-\frac{\partial V\left(\tau_{s-1}\right)}{\partial a}\right|+\left|\frac{\partial V\left(\tau_{s}\right)}{\partial a}-\frac{\partial V\left(t_{0}\right)}{\partial a}\right|
$$

Calcoliamo ora un limite superiore per un generico dei termini che compaiono a secondo membro di (93). Si ha infatti:

$$
\begin{aligned}
\mid \frac{\partial V\left(\tau_{s}\right)}{\partial a} & -\frac{\partial V\left(\tau_{s-1}\right)}{\partial a}|\leq| \frac{\partial V}{\partial a}\left(J\left(\tau_{s}\right), w\left(\tau_{s}\right), a\left(\tau_{s}\right)\right)- \\
& -\frac{\partial V}{\partial a}\left(J\left(\tau_{s-1}\right)\left(w\left(\tau_{s-1}\right)+\omega\left(\tau_{s-1}\right)\left(\tau_{s}-\tau_{s-1}\right), a\left(\tau_{s-1}\right)\right) \mid+\right. \\
+ & \left|\frac{\partial V}{\partial a}\left(J\left(\tau_{s-1}\right), w\left(\tau_{s-1}\right)+\omega\left(\tau_{s-1}\right)\left(\tau_{s}-\tau_{s-1}\right), a\left(\tau_{s-1}\right)\right)-\frac{\partial V}{\partial a}\left(J\left(\tau_{s-1}\right), w\left(\tau_{s-1}\right), a\left(\tau_{s-1}\right)\right)\right| .
\end{aligned}
$$

Ora l'nltimo termine è nullo per la periodicità di $\frac{\partial V}{\partial a}$. Quanto al primo indicato con $M, A, Q$ rispettivamente al massimo di $\left(\frac{\partial V}{\partial a \partial v}\right),\left(\frac{\partial^{2} V}{\partial a \partial J}\right), \frac{\partial^{2} V}{\partial a^{2}}$ per $t$ in $\left(0, t^{\prime}\right), J$ in $J_{0}-\eta, J_{0}+\eta$ e per qualunque $w$ si ha:

$$
\begin{gathered}
\left|\frac{\partial V}{\partial a}\left(J\left(\tau_{s}\right), w\left(\tau_{s}\right), a\left(\tau_{s}\right)\right)-\frac{\partial V}{\partial a}\left(J\left(\tau_{s-1}\right), w\left(\tau_{s-1}\right)+\omega\left(\tau_{s-1}\right)\left(\tau_{s}-\tau_{s-1}\right), a\left(\tau_{s-1}\right)\right)\right| \leq \\
\leq M_{a}\left|w\left(\tau_{s}\right)-w\left(\tau_{s-1}\right)-\omega\left(\tau_{s-1}\right)\left(\tau_{s}-\tau_{s-1}\right)\right|+A\left|J\left(\tau_{s}\right)-J\left(\tau_{s-1}\right)\right|+ \\
+Q\left|a\left(\tau_{s}\right)-a\left(\tau_{s-1}\right)\right| .
\end{gathered}
$$

Ora indicando con $K$ un valore maggiorante in $\left(0, t^{\prime}\right)$ e $J_{0}-\eta, J_{0}+\eta$ per la grandezza finora indicato con questo simbolo e ricordando la (22)

$$
\begin{gathered}
\left|J\left(\tau_{s}\right)-J\left(\tau_{s-1}\right)\right| \leq M_{a} a_{\max }^{\prime}\left(\tau_{s}-\tau_{s-1}\right) \\
\mid a\left(\tau_{s}\right)-a\left(\tau_{s-1} \mid \leq a_{\max }^{\prime}\left(\tau_{s}-\tau_{s-1}\right)\right. \\
\left|v\left(\tau_{s}\right)-w\left(\tau_{s-1}\right)-\omega\left(\tau_{s-1}\right)\left(\tau_{s}-\tau_{s-1}\right)\right| \leq\left(\frac{K+H M_{a}}{\omega_{\min }}+A\right) a_{\max }^{\prime}\left(\tau_{s}-\tau_{s-1}\right) .
\end{gathered}
$$

Quindi si ha:

$$
\left|\frac{\partial V\left(\tau_{s}\right)}{\partial a}-\frac{\partial V\left(\tau_{s-1}\right)}{\partial a}\right| \leq\left(M_{a} \frac{K+H M_{a}}{\omega_{\min }}+2 M_{a} A+Q\right) a_{\max }^{\prime}\left(\tau_{s}-\tau_{s-1}\right) .
$$

Quindi si ha sostituendo in (94)

$$
\left|\frac{\partial V\left(\tau_{r}\right)}{\partial a}\right| \leq\left(M_{a} \frac{\left(K+H M_{a}\right)}{\omega_{\min }}+2 M_{a} A+Q\right) a_{\max }^{\prime} t^{\prime}
$$


Perciò tornando alla (91) abbiamo:

$$
\begin{aligned}
& \left|\int_{\tau_{r}}^{\tau_{r+1}}\left(\sigma(t)-J\left(\tau_{r}\right)\right) d t\right| \leq\left(\frac{M_{a}\left(K+H M_{a}\right)}{\omega_{\min }}+2 M_{a} A+Q\right) \frac{a_{\text {max }}^{\prime 2}}{\omega_{\min }} t^{\prime}\left(\tau_{r+1}-\tau_{r}\right)+ \\
& +\int_{\tau_{r}}^{\tau_{r}+\hat{M}_{a}} \frac{a_{\text {max }}^{\prime \prime}\left(t-\tau_{r}\right)^{2}}{2} d t+Z a_{\text {max }}^{z_{2}} \int_{\tau_{r}}^{\tau_{r+1}}\left(t-\tau_{r}\right)^{2} d t
\end{aligned}
$$

$$
\begin{gathered}
\left|\int_{\tau_{r}}^{\tau_{r+1}}\left(J(t)-J\left(\tau_{r}\right)\right) d t\right| \leq\left(\frac{M_{a}\left(K+H M_{a}\right)}{\omega_{\min }}+2 M_{a} A+Q\right) \frac{a_{\text {max }}^{\prime 2}}{\omega_{\min }} t^{\prime}\left(\tau_{r+1}-\tau_{r}\right)+ \\
\quad+\frac{M_{a} a_{\max }^{\prime \prime}\left(\tau_{r+1}-\tau_{r}\right)^{3}}{6}+\frac{Z a_{\max }^{\prime 2}\left(\tau_{r+1}-\tau_{r}\right)^{3}}{3} \leq \\
\leq\left[\left(\frac{M_{a}\left(K+H M_{a}\right)}{\omega_{\min }}+2 M_{a} A+Q+\frac{Z}{3}\right) \frac{a^{\prime 2} \max }{\omega_{\min }} t^{\prime}+\frac{M_{a} a^{\prime \prime}}{6 \omega_{\max }} t^{\prime}\right]\left(\tau_{r+1}-\tau_{r}\right) .
\end{gathered}
$$

Quindi si ha:

(101)

$$
\begin{gathered}
\left|\int_{\tau_{r}}^{\tau_{r+1}}\left(\omega(J, a)-\omega\left(J\left(t_{0}\right), a\right)\right) d t\right| \leq \\
\leq\left(\frac{H M_{a}\left(K+H M I_{a}\right)}{\omega_{\min }}+\frac{2 H M_{a} A+H Q}{\omega_{\min }}+\frac{4}{3} \frac{Z H}{\omega_{\min }}\right) a^{\prime 2}{ }_{\max } t^{\prime}\left(\tau_{r+1}-\tau_{r}\right)+ \\
+\frac{L}{\omega_{\min }} \alpha^{\prime 2}{ }_{\max } t^{\prime}\left(\tau_{r+1}-\tau_{r}\right)+\frac{2}{3} M_{a} H \frac{a_{\text {max }}^{\prime \prime}}{\omega_{\min }} t^{\prime}\left(\tau_{r+1}-\tau_{r}\right)= \\
=m a^{\prime 2}{ }_{\max } t^{\prime}\left(\tau_{r+1}-\tau_{r}\right)+n a^{\prime \prime}{ }_{\max } t^{\prime}\left(\tau_{r+1}-\tau_{r}\right) .
\end{gathered}
$$

Essendo:

$$
\begin{gathered}
m=\frac{H M_{a}\left(K+H M_{a}\right)}{\omega_{\min }}+\frac{2 H M_{a} A+H Q}{\omega_{\min }}+\frac{4}{3} \frac{Z H}{\omega_{\min }}+\frac{L}{\omega_{\min }} \\
n=\frac{2}{3} \frac{M_{a t} H}{\omega_{\min }} .
\end{gathered}
$$

Si ha poi:

$$
\begin{aligned}
& \left|\iint_{\tau_{n-1}}^{t^{\prime}} \omega(J(t), a)-\omega\left(J\left(t_{0}\right), a\right)\right| d t \mid \\
& \left|\iint_{\tau_{n-1}}^{t^{\prime}}\right| \omega(J, a)-\omega\left(J\left(\tau_{n-1}\right), a\right)|d t|+\left|\int_{\tau_{n-1}}^{t} \omega\left(J\left(\tau_{n-1}\right), a\right)-\omega\left(J\left(t_{0}\right), a\right) d t\right| \leq \\
& \leq H \int_{\tau_{n-1}}^{t}\left|J(t)-J\left(\tau_{n-1}\right)\right| d t+\frac{H Z a^{\prime \prime}{ }_{\max } t^{\prime}}{\omega_{\min }} \int_{\tau_{n-1}}^{t^{\prime}} d t+\frac{M_{a} H}{2} \frac{a^{\prime \prime}{ }_{\max } t^{\prime}}{\omega_{\min }} \int_{\tau_{n-1}}^{t^{\prime}} d t \leq \\
& \leq \frac{H M \Delta_{m} a}{\omega_{\min }}+\left|m a^{\prime 2}{ }_{\max } t^{\prime}+n a^{\prime \prime}{ }_{\max } t^{\prime}\right|\left(t^{\prime}-\tau_{n-1}\right) .
\end{aligned}
$$


Allora sommando la (104) con le (101) e (76) si ha:

$$
\left|\int_{0}^{t} \omega(J, a)-\omega\left(J\left(t_{0}\right), a\right) d t\right| \leq \frac{2 H M}{\omega_{\operatorname{mix}}} \Delta_{m} a+m\left(a_{\text {max }}^{\prime} l^{\prime}\right)^{2}+n \alpha_{\text {max }}^{\prime \prime} t^{2} .
$$

e da questa formula si ottiene facilmente, aggiungendo $\left(\bar{Z} \Delta_{m} a+A \Delta_{m} a^{\prime}\right) V\left(a, t^{\prime}\right)+$ $+A \Delta_{m} a$ ) la cercata espressione per il limite superiore dell' errore commesso nel sostituire alla soluzione esatta del sistema (65) la soluzione approssimata. Come si vede a parte il termine in $\Delta_{m} a$, ohe è di solito piccolissimo, l' errore dipende $\mathrm{da}\left(a_{\max }^{\prime} t^{\prime}\right)^{2}$ e $a_{\text {max }}^{\prime \prime} t^{\prime 2}$. Ora se la $a$ variasse in modo lineare $a_{\text {max }}^{\prime \prime} t^{2}$ sarebbe nulla mentre $\alpha_{\max }^{\prime} t^{\prime}$ sarebbe l'ineremento che subisce la $a$ nell' intervallo $\left(0, t^{\prime}\right)$.

Perciò in questo caso si può affermare che la nostra integrazione approssimata è valida nell'intervallo in cui si può ritenere infinitesimo il quadrato degli incrementi di $a$. Se invece $a$ non varia in modo lineare non è possibile un enunciato così semplỉce del nostro restultato. Si pnò però osservare che essendo $\Delta_{m} a^{\prime}$ molto piccolo nell' intervallo $\left(0, t^{\prime}\right)$ si può presumere $\alpha_{\text {max }}^{\prime \prime} t^{\prime 2}$ dell' ordine di $\left(\alpha_{\max }^{\prime} t^{\prime}\right)^{2}{ }^{\left({ }^{1}\right)}$, e perciò si può ancora enunciare il teorema nel modo sopra indicato.

Comunque per più esattezza la fermula (105) serve in ogni caso per verificare entro quale intervallo la soluzione approssimata è sostituibile a quella esatta. Prima di lasciare la questione, vogliamo notare che, come scende anche dalla nostra dimostrazione, sarebbe stato possibile trovare un limite superiore più basso di quello calcolato, ma esso sarebbe risultato assai più incomodo nelle applicazioni. Osserviamo poi che per esprimere l'errore commesso supponiamo noto il modo di variare di $a$ ossia di conoscere $a_{\max }^{\prime}$. Ma ciò è necessario altrimenti non sarebbe possibile il calcolo di $\int_{0}^{t^{\prime}} \omega\left(J\left(t_{0}\right), a\right) d t(\%)$.

9. Calcolo approssimato del numero delle oscillazioni. - Se il metodo d'integrazione approssimata delle nostre equazioni è valido solo per un intervallo di tempo che possiamo dire ristretto, rispetto all' intervallo in eui la $J$ si

(1) Questa presunzione si può giustificare in base al fatto che $\Delta a^{\prime}, \Delta a$ sono dello stesso ordine di grandezza $e \frac{a^{\prime}}{a^{\prime} \max }, \frac{a^{\prime \prime}}{a^{\prime \prime} \max }$ sono in $\left(0, t^{\prime}\right)$ molto vicine all' unità. Allora è facile de* durre $a^{\prime \prime}$ max dell' ordine di $a^{\prime 2} \max$ cioè $a^{\prime \prime}{ }_{\max } t^{\prime 2}$ dell" ordine di $\left(a_{\text {max }}^{\prime} t^{\prime}\right)^{2}$.

(2) $\mathrm{E}$ poi ovvio che la nostra integrazione vale per $t^{\prime}$ nell'intervallo $(0, h)$ in cui $\left|J-J_{0}\right|<\eta$. 
può ritenere costante, è possibile però partendo dalla (67) ottenere per certe grandezze delle approssimazioni valide per tutto l'intervallo in cui $\Delta J$ si può ritenere trascurabile.

Noi calcoleremo con approssimazione più che sufficiente per tutti i bisogni della pratica, il numero delle oseillazioni che subisce il nostro sistema, intendendo per numero delle oscillazioni il numero delle volte in cui $w$ assume valori interi, ossia in sostanza la $w$ stessa, ma questa volta interessa che l'errore sia piccolo rispetto alla $w$ non come nel paragrafo precedente rispetto all' unità. Questa ricerca può avere intẹresse per esempio se si volesse conoscere quante volte un pianeta è passato in un certo tempo al perielio.

Come è noto la $w$ vale:

$$
w=\int_{0}^{t^{\prime}} \omega(J, a) d t+\int_{0}^{t^{\prime}} a^{\prime} w_{a} d t
$$

Mentre il valore approssimato ehe noi proponiamo per il calcolo delle oscillazioni sarà

$$
w^{\prime}=\int_{0}^{t \prime} \omega\left(J_{0}, \alpha\right) d t
$$

Quindi l'errore relativo commesso $\lambda$ vale:

$$
\lambda=\frac{\int_{0}^{t^{\prime}} \omega(J, a)-\omega\left(J_{0}, a\right) d t+\int_{0}^{t^{\prime}} a^{\prime} w_{a^{\prime}} d t}{\left|\int_{0}^{t^{\prime}} \omega(J, a) d t+\int_{0}^{t^{\prime}} a^{\prime} w_{a} d t\right|} .
$$

Ossia :

$$
\lambda=1-\frac{\int_{0}^{t^{\prime}} \omega\left(J_{0}, a\right) d t}{\int_{0}^{t^{\prime}} \omega\left(J_{0}, \alpha\right) d t+\int_{0}^{t^{\prime}}\left(\omega(J, a)-\omega\left(J_{0}, \alpha\right)\right) d t+\int_{0}^{t^{\prime}} a^{\prime} w_{n} d t .} .
$$

Ora è :

$$
\left|\int_{0}^{t} a^{\prime} w_{a} d t\right|<\left(\bar{Z} \Delta_{m} a+A \Delta_{m} a^{\prime}\right) V(a, t)+A \Delta_{m} a
$$

Si ha poi applicando il teorema della media $e$ indicando con $\bar{t}$ un va- 
lore del tempo intermedio fra $\left(0, t^{\prime}\right)$

$$
\begin{gathered}
\left|\int_{0}^{t^{\prime}}\left(\omega(J, a)-\omega\left(J_{0}, a\right)\right) d t\right|=\left|\int_{0}^{t^{\prime}} \omega\left(J_{0}, a\right)\left(1-\frac{\omega(J, a)}{\omega\left(J_{0}, a\right)}\right) d t\right|= \\
=\left|\int_{0}^{t^{\prime}} \omega\left(J_{0}, a\right) d t\right|\left|\frac{\omega(J(\bar{t}), a(\bar{t}))-\omega\left(J_{0}, a(\bar{t})\right)}{\omega\left(J_{0}, \alpha(\bar{t})\right)}\right| \leq\left|\int_{0}^{t^{\prime}} \omega\left(J_{0}, a\right) d t\right| \frac{H}{\omega_{\min }}\left|J(\bar{t})-J_{0}\right| \leq \\
\leq \int_{0}^{t^{\prime}} \omega\left(J_{0}, a\right) d t\left|\frac{H}{\omega_{\min }}\left[Z \Delta_{m} a+M_{a} \Delta_{m} a^{\prime}\right] V(a, t)+\frac{H M}{\omega_{\min }} \Delta_{m} a\right| .
\end{gathered}
$$

Ossia :

(111)

$$
\lambda=1-\frac{1}{1+\frac{H}{\omega_{\min }}\left(Z \Delta_{m} a+M_{a} \Delta_{m} a^{\prime}\right) V\left(a, t^{\prime}\right)+\frac{\left(Z \Delta_{m} a+A \Delta_{m} a^{\prime}\right) V\left(a, t^{\prime}\right)+A \Delta_{m} a}{\int_{0}^{t^{\prime}} \omega\left(J l_{0}, a\right) d t}+\frac{H M_{a} \Delta_{m} a}{\omega_{\min }}}
$$

Come si vede subito con una semplice riduzione questo limite superiore per $\lambda$ è dell'ordine di $\Delta_{n} a$ e $\Delta_{n} a^{\prime}$. E perciò se queste grandezze sono molto piccole risulta subito che $\lambda$ è piccolo, restando così dimostrato il nostro asserto.

\section{CAPITOLO III.}

10. Generalità sui sistemi a due parametri variabili. - Riprendiamo ora il caso in cui il nostro sistema meccanico abbia due parametri $a$ e $b$. Supporremo che $a$ vari al solito in maniera tale che $\Delta a$ e $\Delta a^{\prime}$ siano molto piccoli cioè in modo da avvicinarsi a variare in maniera infinitamente lenta e graduale, mentre sul modo di variare di $b$ non facciamo per ora aleuna ipotesi. Allora vogliamo dimostrare che la differenza fra $J$ e la $y$ definita nel capitolo primo, è nulla se $a$ varia in modo infinitamente lento e graduale ed in ogni caso è possibile dare un limite superiore per la differenza $|J-y|$ in modo da poter verificare se la $y$ è sostituibile alla $J$ con sufficiente approssimazione.

Percio riprendiamo la formula (14) del capitolo primo:

$$
|J-y| \leqq\left|\int_{0}^{t^{\prime}} a^{\prime} J_{a} d t\right|+\left|\int_{0}^{t^{\prime}} b^{\prime}\left(J_{b}-\bar{J}_{b}\right) d t\right| .
$$


E poichè $J_{b}$ è Lipschitziana possiamo scrivere:

$$
\left|J_{b}-\bar{J}_{b}\right| \leq T|J-y|
$$

Essendo $T$ il massimo di $\frac{\partial J_{b}}{\partial J}$ per $t$ in $\left(0, t^{\prime}\right)$ e $J$ compreso fra $y_{\max }+\eta$, $y_{\min }-\eta$ e per ogni $w$ : Naturalmente come nel capitolo primo supponiamo $\left(0, t^{\prime}\right)$ compreso nell' intervallo $(0, h)$ in cui $|J-y|$ è inferiore a $\eta$.

Si ha allora:

$$
|J-y| \leq I\left(t^{\prime}\right)+T \int_{0}^{t^{\prime}}\left|b^{\prime} \| J-y\right| d t
$$

Dove $I\left(t^{\prime}\right)$ è il limite superiore già calcolato per $\left|\int_{0}^{t^{\prime}} a^{\prime} J_{a} d t\right|$ ossia $M_{a} \Delta_{m} a+$ $+\left(Z \Delta_{m} a+M_{n} \Delta_{m} \alpha^{\prime}\right) V\left(a, t^{\prime}\right)+Z^{\prime} \Delta_{n} a V(b, t)$

Per trovare il limite superiore cercato si consideri la funzione soddisfacente all' equazione:

$$
V=I\left(t^{\prime}\right)+T \int_{0}^{t^{\prime}}\left|b^{\prime}\right| V d t
$$

Noi dimostreremo anzitutto che $V$ è maggiore di $|J-Y|$. A questo scopo si sottragga la (115) dalla (114). Si ha:

$$
|J-y|-V \leq T \int_{0}^{t^{\prime}}\left(b^{\prime} \mid(|J-Y|-V) d t\right.
$$

Ora per $t=0$ è $J-y=0$ mentre $V$ vale $I(0)=M_{a} \Delta_{m} a$. Allora per $t^{\prime}=0,|J-y|-V<0$ e per continuità resterà tale in un intorno $(0, \delta)$. E facile vedere che 1 'intorno $(0, \delta)$ coincide con $\left(0, t^{\prime}\right)$. Difatti se fosse $\delta<t^{\prime}$ sarebbe per $t=\delta,|J-y|-V=0$. Ma essendo in $(0, \delta),|J-y|-V$ negativo il secondo membro sarebbe per $t=\delta$ negativo, mentre il primo sarebbe nullo il che è assurdo. Segue perciò $|J-y|<V$ in tutto $\left(0, t^{\prime}\right)$.

Calcoliamo ora $V$ in base alla sua definizione (115). Derivando questa equazione si ha:

$$
\frac{d V}{d t}=\frac{d I}{d t}+T\left|b^{\prime}\right| V=\left(Z \Delta_{m} a+M_{s} \Delta_{m} a^{\prime}\left|\frac{d a}{d t}\right|+Z^{\prime} \Delta_{m} a\left|\frac{d b}{\bar{d} t}\right|+T\left|b^{\prime}\right| V\right.
$$


e ntegrando questa equazione differenziale si ha:

$$
\begin{aligned}
V= & e^{T \int\left|b^{\prime}\right| d t} \int_{0}^{t^{\prime}} e^{\int_{0}^{t} T\left|b^{\prime}\right| d t}\left[\left(Z \Delta_{m}^{\prime} a+M_{a} \Delta_{m} a^{\prime}\right)\left|\frac{d a}{d t}\right|+Z^{\prime} \Delta_{m} a\left|\frac{d b}{d t}\right|\right] d t+V(0) \leq \\
& \leq e^{T V\left(b, t^{\prime}\right)}\left(\left\{Z \Delta_{m} a+M_{a} \Delta_{m} a^{\prime}\right\} V\left(a, t^{\prime}\right)+Z^{\prime} \Delta_{m} a V\left(b, t^{\prime}\right)+M_{a} \Delta_{m} a\right) .
\end{aligned}
$$

Quindi:

$$
\begin{aligned}
|J-y| \leq e^{T V\left(b, t^{\prime}\right)}\left(\left\{Z \Delta_{m} a+M_{a} \Delta_{m} a^{\prime}\right\} V\left(a, t^{\prime}\right)+\right. \\
\left.+Z^{\prime} \Delta_{m} a V\left(b, t^{\prime}\right)+M_{a} \Delta_{m} a\right)=e^{T V\left(b, t^{\prime}\right)} I\left(t^{\prime}\right) .
\end{aligned}
$$

Da questa formula è facile anzitutto verificare in quale intervallo $\left(0, t^{\prime}\right)$ resta sicuramente $|J-y|$ inferiore a $\eta$ perchè basta verificare per le ragioni esposte nel capitolo primo se in un certo intervallo è inferiore a $\eta$ il secondo membro della formula in discorso. Naturalmente si vede subito che a parita di $V\left(a, t^{\prime}\right), V\left(b, t^{\prime}\right)$ questo intervallo è tanto più ampio quanto più piccolo è $\Delta_{m} \alpha$ e $\Delta_{m} \alpha^{\prime}$ anzi se. la $\alpha$ varia in maniera infinitamente lenta e graduale la differenza fra $J$ e $y$ è nulla in qualunque intervallo $\left(0, l^{\prime}\right)$ (') finito o infinito in cui $V\left(a, t^{\prime}\right), V\left(b, t^{\prime}\right)$ restano finite. Questo resultato si ottiene con considerazioni analoghe a quelle svolte nel paragrafo 4 del capitolo primo.

11. Osservazioni critiche. - Dobbiamo ora agginngere alcune osservazioni sulla portata pratica del nostro resultato.

Si noterà anzitutto che la formula (117) è applicabile se $V\left(a, t^{\prime}\right), \quad V\left(b, t^{\prime}\right)$ sono finite. Ora affinchè $V\left(a, t^{\prime}\right)$ abbia un valore finito è abbastanza grande essendo $\Delta_{m} a$ molto piccolo, deve essere grande il numero di periodi compresi in $\left(0, t^{\prime}\right)$. Ma allora salvo casi speciali, dovrebbe essere $\Delta b$ molto piccolo altrimenti $V\left(b, t^{\prime}\right)$ diventerebbe enorme e la nostra limitazione non sarebbe appli. cabile praticamente.

Sembrerebbe da ciò che in sostanza per applicare la (117) fosise neces. sario ammettere che la $b$ vari in modo analogo alla $a$ e che perciò il nostro resultato non avesse alcun valore perchè si riducesse al caso di un sistema in cui vi sono due parametri variabili come $a$. Si deve però notare che per avere $V\left(b, t^{\prime}\right)$ finito, si deve per quanto precede supporre in generale $\Delta b$ piccolo, ma non vi è alcuna ipotesi da farsi su $\Delta b$. Pereiò si può dire che il nostro resultato vale se $b$ varia in una maniera che si avvicina ad essere

(1) S'intende compreso in $\left(0, h^{\prime}\right)$. 
infinitamente lenta, ma non si avvicina affatto ad essere graduale. Questioni di questo genere si presentano nella meccanica celeste.

Altra obbiezione che si può fare al nostro resultato è che la $y$ viene ricavata da una equazione differenziale in cui appare $\bar{J}_{b}$ che dipende da $w$ la quale è incoynita fino a che non si sappia integrare tutto il sistema (5). Perciò il nostro resultato è applicabile quando per altra via si conosce la $w(t)$ oppure il caso in cui si possa esprimere $w$ come funzione di $b$ perchè allora basta integrare l' equazione

$$
\frac{d y}{d t}=-b^{\prime} \bar{\jmath}_{b}
$$

in cui la sola incognita è la $y$. Di questo caso vedremo una applicazione alla meccanica celeste.

Poi il nostro resultato può avere interesse qualora si vogliono conoscere alcune limitazioni per la $J$. In questo caso può essere facile trovare un limite superiore per $y$ in base alla (13) e da questa risalire ad una limitazione per $J$.

Speriamo in ricerche future di mostrare alcune applicazioni delle for* mule ottenute in questo capitolo. 